\title{
A Multimaterial Extension to Subzonal Reconstruction
}

\author{
D. P. Starinshak ${ }^{\mathrm{a}}$, J. M. Owen ${ }^{\mathrm{b}}$ \\ ${ }^{a}$ Lawrence Livermore National Laboratory, AX Division, M/S L-38, P.O. Box 808, \\ Livermore, CA 94550 \\ ${ }^{b}$ Lawrence Livermore National Laboratory, AX Division, M/S L-38, P.O. Box 808, \\ Livermore, CA 94550
}

\begin{abstract}
We present a new algorithm for reconstructing material-dependent subzonal information based on staggered primary/dual-mesh remapping of materialdependent conserved quantities. The algorithm is appropriate in the context of geometric, intersection-based overlay remapping methods, with specific application to staggered, total energy conserving, multi-material Lagrangian hydrodynamics schemes that discretize material masses on subzonal mesh elements. Our new approach avoids direct remapping of material-dependent subzonal variables; instead, the spatial profile of each variable is reconstructed using a combination of material-dependent zone (primary mesh) information, material-independent node (dual mesh) information, and discrete interface-reconstructed material concentration information. Conservation and convergence properties of the new algorithm are established through several challenging multi-material remapping and hydrodynamics tests.
\end{abstract}

Keywords: Remapping, ALE methods

\section{Introduction}

In [23], we outlined an algorithm for conservatively reconstructing subzonal information using staggered data at zones (primary mesh) and nodes (dual mesh). Because the number of subzonal elements in the mesh exceeds the total number of zone and node elements, reconstruction takes the form

Email addresses: starinshak1@llnl.gov (D. P. Starinshak), mikeowen@llnl.gov (J. M. Owen)

Preprint submitted to Elsevier

November 23, 2015

(C) 2015. This manuscript version is made available under the Elsevier user license http://www.elsevier.com/open-access/userlicense/1.0/ 
of a least-squares optimization procedure. Conservation is achieved by enforcing linear equality constraints at each zone and node.

This work is motivated by staggered, compatible [5] Arbitrary LagrangianEulerian (ALE) methods that employ an overlay-based (also known as intersection, interpolation, or geometric) remap. In the staggered, compatible framework, mass is discretized onto subzonal elements to ensure consistent representations of mass at both zones and nodes. Conservation of mass, linear momentum, and energy is a natural consequence of this discretization.

In [15], the authors propose a separate method for remapping subzonal data. State variables are collocated to subzonal elements and remapped from subzones to subzones. We initially proposed the reconstruction algorithm in [23] to alleviate the expense of computing subzone-subzone intersections for each remap. The computational cost of an overlay remap scales with the number of element intersections. Timing data and scaling arguments in [23] indicate that staggered overlay remapping (i.e. computing zone-zone and node-node intersections) followed by subzonal reconstruction outperforms direct subzonal remapping in terms of computational efficiency for $2 \mathrm{D}$ polygonal meshes.

A second motivation for pursuing subzonal reconstruction over direct subzonal remapping is the problem of remapping multimaterial data. The direct subzonal remap described in [15] and applied in [14] is only applicable for single-material problems. Extending the methodology to multi-material data in an efficient manner is not straightforward; at the very least, a subzonal remap requires a multi-material model that is discretized onto subzonal elements. In the context of multi-material ALE calculations, this implies storing discrete volume and/or mass fraction data at subzones as well as performing interface reconstruction at a sub-grid scale. In our experience, most multi-material hydro codes discretize material concentrations on zone elements. Adapting such codes to handle the higher memory and complexity requirements of a fully subzonal multi-material discretization is not optimal - rather we prefer to adapt our remap algorithm to handle zone centered material fractions.

In this paper, we describe a multimaterial extension to the subzonal reconstruction algorithm. The extension is conservative and requires minimal modification of the host code to operate. We consider a volume-of-fluid (VOF) multimaterial model with volume fraction data discretized onto zonal elements [1]. In principle, the algorithm may be adapted to operate within the moment-of-fluid (MOF) multimaterial framework as well with little mod- 


\section{ification.}

Note that the problem of remapping multimaterial subzonal masses in a conservative, monotonic, and efficient manner is not confined to overlaybased remaps. Similar problems arise in advective/flux-based remaps as well. The availability of numerical mass fluxes simplifies the problem somewhat. Interested readers may consider [17] for advective remapping strategies for staggered, compatible discretizations.

Hybrid methods have also been developed recently to bridge the gap between overlay and advective remaps. Numerical mass fluxes may be obtained from intersection data and limited in intelligent ways to promote conservation, monotonicity, and accuracy. In the context of this work, we point the reader toward [10, 11].

The paper is organized as follows: section 2 describes the compatible subzonal discretization of hydrodynamic variables; section 3 defines the overlay procedure for remapping single- and multi-material data; section 4 details the three-stage multimaterial subzonal reconstruction algorithm; and section 5 demonstrates the new algorithm's performance a series of pure remapping and ALE hydrodynamics test problems.

\section{Background}

\subsection{Subzonal Discretization}

Our main application in this paper is the use of a geometric overlay-based remap with the spatially-staggered, Lagrangian-frame algorithm for hydrodynamics outlined in [5]. The Lagrangian hydrodynamic discretization of [5] derives a number of important properties - for instance, conservation, generality to unstructured grids, and improved accuracy - from the discretization of state variables on subzonal mesh elements.

Each zone is subdivided into subzonal elements, often referred to as corners, indexed here by $c$. Each corner is a polyhedron in 3D or a quadrilateral in $2 \mathrm{D}$ and corresponds to a unique neighboring zone-node pair $\left(z_{c}, n_{c}\right)$ on an arbitrary, unstructured mesh. The vertices of corner $c$ consist of the centroid of zone $z_{c}$, the position of node $n_{c}$, and the centers of each of the faces and edges of $z_{c}$ that touch $n_{c}$ (see fig. 11).

Element volumes for zones $V_{z}$ and nodes $V_{n}$ are defined by summing neighboring corner volumes $V_{c}$ :

$$
V_{z}=\sum_{c(z)} V_{c} \quad \text { and } \quad V_{n}=\sum_{c(n)} V_{c} .
$$


Here $c(z)$ denotes the set of corners inside zone $z$, and $c(n)$ denotes the set of corners touching node $n$. The union of each corner element in $c(n)$ defines the mesh element associated with node $n$ (see fig. 2). A corner can thus be thought of as the intersection of zone element $z_{c}$ on the primary mesh and node element $n_{c}$ on the dual mesh.
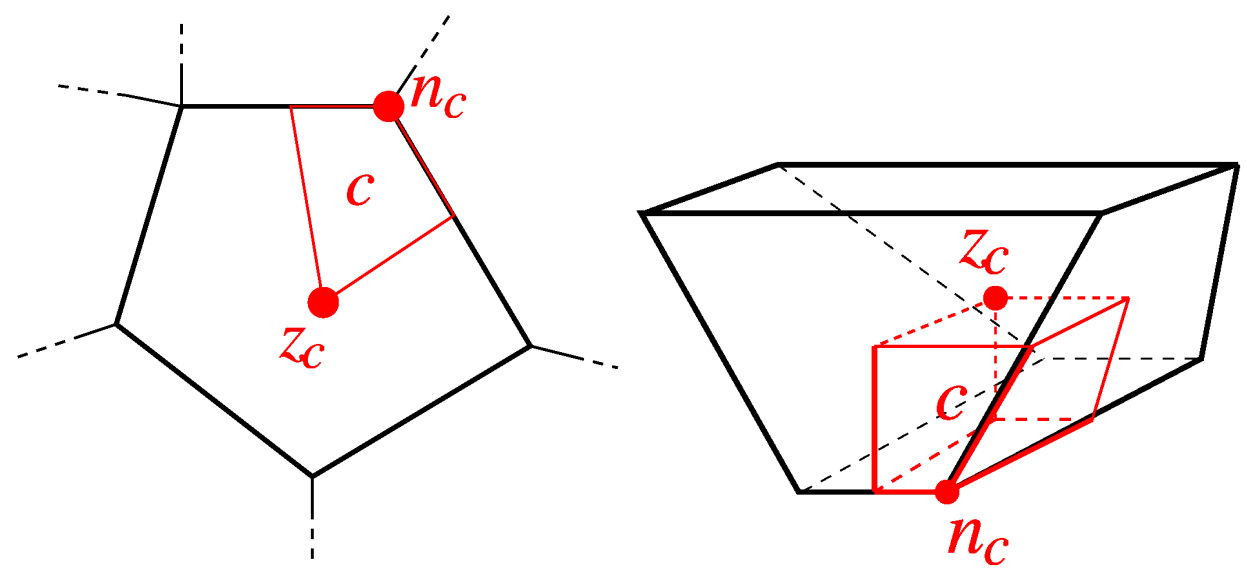

Figure 1: Corner subvolumes ( $c$, in red) of a 2D (left) and 3D (right) zone.

\subsection{Subzonal Densities and Compatible Hydrodynamics}

The staggered, compatible discretization for conservative hydrodynamics places kinematic variables of velocity and kinetic energy at nodes and thermodynamic variables of internal energy at zone centers. In order to facilitate Lagrangian definitions of the mass on both zones and nodes, the fluid mass is stored on the subzonal corners. Discrete zonal and nodal masses are then naturally defined as coarsened representations of these subzonal corner masses on their respective element volumes:

$$
m_{z}=\sum_{c(z)} m_{c} \quad \text { and } \quad m_{n}=\sum_{c(n)} m_{c}
$$

where $m_{z}, m_{n}$, and $m_{c}$ denote discrete masses centered at zone $z$, node $n$, and corner $c$ respectively. Again, the notation " $\mathrm{c}(\mathrm{z})$ " and " $\mathrm{c}(\mathrm{n})$ " is shorthand for "corners within zone $z$ " and "corners around node $n$ ", respectively. The corresponding densities satisfy

$$
\rho_{\alpha}=m_{\alpha} / V_{\alpha}, \quad \alpha=z, n, c .
$$




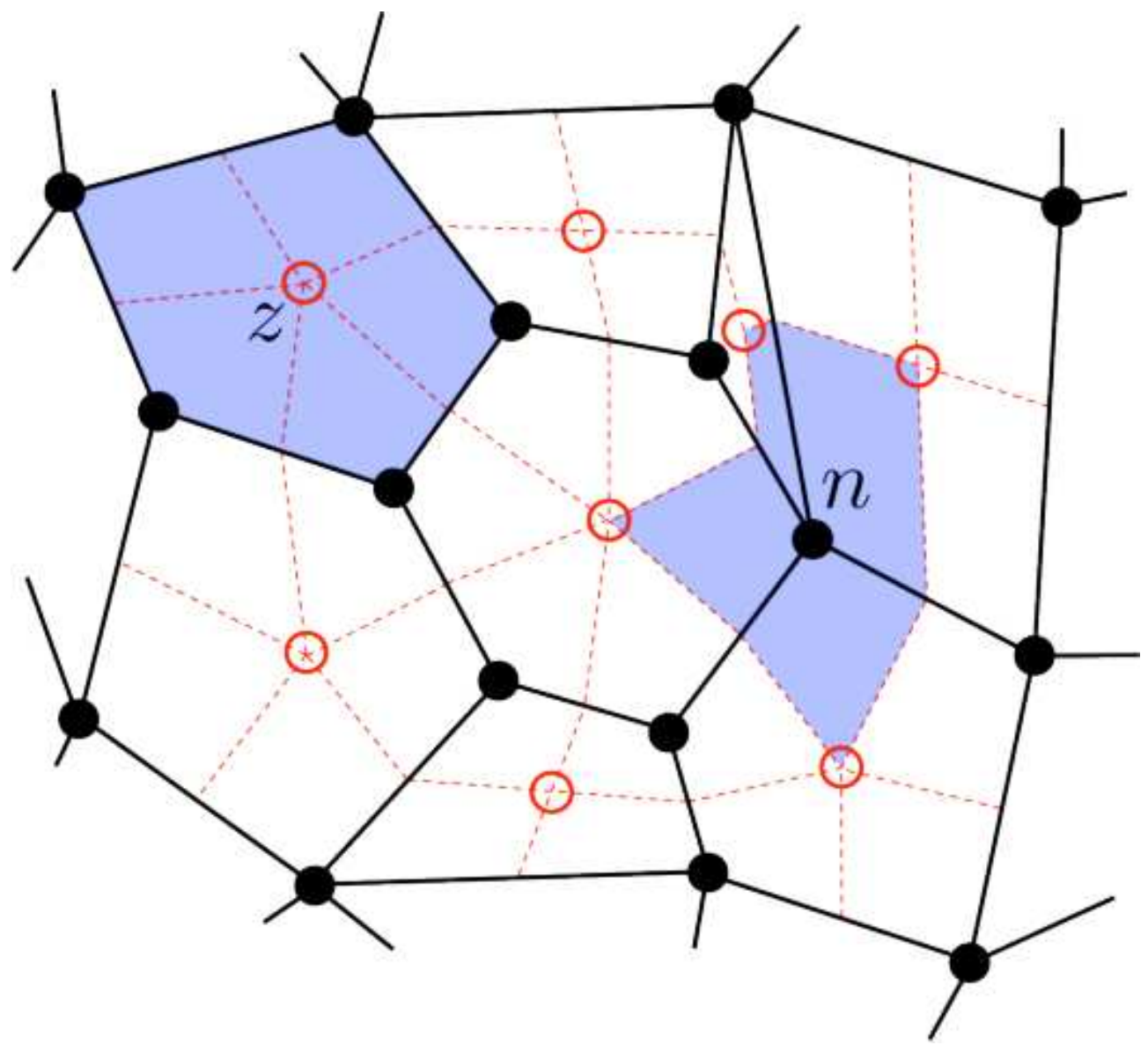

Figure 2: An unstructured polygonal mesh. Zones are outlined by solid black lines with barycenters given by open red circles. Node positions are solid black circles. Dashed red lines indicate corner subelements within each zone. Control volumes for a zone $z$ and node $n$ are highlighted.

\subsection{Multimaterial Discretization}

We consider the volume-of-fluid (VOF) multimaterial model described in [1]. The model describes the $p$-th fluid component by its specific mass density and specific internal energy density. Each fluid component is assumed to have the same velocity. Note that internal energy does not factor into the proposed algorithm; we include it in subsequent discussions for the sake of completeness.

The discrete VOF model is centered on zone elements. The discrete 
material volume fraction for the $p$-th fluid component in zone $z$ is denoted $f_{z}^{p}$ and satisfies the no-void condition

$$
\sum_{p} f_{z}^{p}=1
$$

A zone is considered mixed if $f_{z}^{p}$ is nonzero for multiple components $p$.

The discrete model is memory-efficient in that mixed-material physics is only resolved at the zone scale; no additional information is discretized onto subzones. The implication of this is that each subzonal element contained in a mixed-material zone is assumed to have identical material concentrations (i.e. $f_{c}^{p}=f_{z_{c}}^{p}$ ).

As in the single-material case, per-material mass densities are also discretized onto subzonal elements. The partial mass density of component $p$ in subzone $c$ is denoted $\rho_{c}^{p}$ and defines the $p$-th mass element by

$$
m_{c}^{p}=f_{z_{c}}^{p} \rho_{c}^{p} V_{c}=\rho_{c}^{p} V_{c}^{p} .
$$

Here $V_{c}^{p}=f_{z_{c}}^{p} V_{c}$ denotes the volume occupied by component $p$ within corner element $c$. As in eq. (2), the per-material masses at each zone and node are local sums of neighboring subzonal masses:

$$
m_{z}^{p}=f_{z}^{p} \rho_{z}^{p} V_{z}=\rho_{z}^{p} V_{z}^{p}=\sum_{c(z)} m_{c}^{p}
$$

and

$$
m_{n}^{p}=\sum_{c(n)} m_{c}^{p}
$$

\section{Overlay Remap Procedure}

The overlay remap problem amounts to mapping discrete data on an arbitrarily-connected mesh (referred to here as the donor mesh) onto another arbitrarily-connected and potentially unrelated mesh (referred to here as the target). The data is conserved with respect to volume elements (i.e. mass, momentum, and energy densities for fluid flow problems) and can be spatially staggered. Zone-centered donor data is mapped to target zones through zonezone intersections; likewise, node-centered data on the donor dual mesh is mapped to target nodes through node-node intersections. Node volumes are defined by eq. (1). 
In this paper, all remapping operations are performed using the overlay tool Overlink [6]. Overlink performs geometric intersections between arbitrary polygonal (polyhedral) mesh elements by subdividing them into triangles (tetrahedra) and computing exact intersections. Overlink computes linear reconstructions of conserved remap variables using neighboring data. The remapped state on the target mesh is formally second-order accurate with respect to the original donor state for smooth initial data. Linear reconstructions are slope limited to ensure monotonicity in the post-remap state.

Multiple materials are handled by discrete zone-centered volume fractions. Overlink constructs geometric interfaces in each mixed zone using neighboring volume fraction data and the ELVIRA algorithm [18]. Interface reconstruction is required by any overlay package to accurately compute intersections. Without geometric interface data, mixed-material zones would be homogeneous with respect to their volume fractions. As a consequence, materials would numerically diffuse into one another thereby creating material mixtures at nonphysical rates.

To ease notation, we will denote the pre-remap (donor) value of a quantity $q$ on element $\alpha$ by $\widetilde{q}_{\alpha}$. Its post-remap value is simply $q_{\alpha}$.

\subsection{Single Material Remap Data}

The subzonal reconstruction algorithm takes post-remap densities at zones and nodes as input. The minimal data passed through the overlay at zones and nodes is summarized:

$$
\begin{aligned}
& \tilde{\rho}_{z} \longrightarrow \rho_{z} \\
& \widetilde{\rho}_{n} \longrightarrow \rho_{n}
\end{aligned}
$$

Additional remap data depends on the problem of interest. (For instance, for compressible hydro calculations, we additionally remap momentum, internal energy, and kinetic energy.) The reconstruction procedure, denoted $\mathcal{R}$, computes conservative subzonal densities using the post-remap zone and node masses: $\rho_{c}=\mathcal{R}\left(m_{z}, m_{n}\right)$.

\subsection{Multimaterial Remap Data}

When multiple materials are present, a richer set of data must be remapped. In addition to the total mass densities at each zone and node, we remap the 
volume fractions $f_{z}^{p}$ and the specific mass densities $\rho_{z}^{p}$ for each material $p$ :

$$
\begin{aligned}
\left(\widetilde{\rho}, \tilde{f}^{p}, \widetilde{\rho}^{p}\right)_{z} & \longrightarrow\left(\rho, f^{p}, \rho^{p}\right)_{z} \\
\widetilde{\rho}_{n} & \longrightarrow \rho_{n}
\end{aligned}
$$

A single reconstruction is performed to compute the total subzonal mass density.

The choice to remap the set of data summarized in eq. (9) is based on a combination of algorithmic needs and code limitations. We note:

- Volume fractions are required data for any such overlay remap. The pre-remap values $\widetilde{f}_{z}^{p}$ inform the interface reconstruction algorithm. Their post-remap values are directly computed by zone-zone intersections.

- Redundant data is remapped at each zone. Although the pre-remap state satisfies

$$
\widetilde{\rho}_{z}=\sum_{p} \widetilde{f}_{z}^{p} \widetilde{\rho}_{z}^{p}
$$

it is not necessarily true after the remap. The reason is because values of $f_{z}^{p} \rho_{z}^{p}$ depend on interface reconstruction, but their sum (which is independently-remapped) does not. (See fig. 3.) The discrepancy in their post-remap values is important information for the multimaterial reconstruction problem. This is described in detail in section 4.1.1.

- Material-dependent nodal densities are not remapped. Doing so would require us to perform interface reconstruction at both primary and dual mesh elements to accurately remap material volumes. The reconstructed interfaces at zones and nodes are not guaranteed to be consistent if they are computed independently. And computing a coupled primary-dual interface may not be feasible for all codes. Of course, by remapping only the total nodal mass density, we enjoy computational savings resulting from remapping fewer dual mesh variables.

\section{Multimaterial Subzonal Reconstruction}

The multimaterial reconstruction procedure is divided into three stages. First is a preprocessing stage; the post-remap total zone and node densities are modified to account for front steepening due to interface reconstruction 
and repaired, as in [16] to promote monotonicity. Second, a total subzonal mass density field is computed using the reconstruction algorithm detailed in [23]. The final stage assigns material concentrations to each subzone using material-dependent post-remap zone densities.

We discuss each stage in greater detail below and summarize the final algorithm in section 4.4.

\subsection{Stage 1: Preprocessing Remapped Data}

Simply applying the reconstruction algorithm $\mathcal{R}\left(m_{z}, m_{n}\right)$ can produce total subzonal densities that are inconsistent with material interfaces and discrete volume fractions. The potential for post-remap zone and node densities to be non-monotonic with respect to one another is also higher resulting from interface reconstruction. A series of pre-processing steps are performed to make the reconstruction stage more robust.

\subsubsection{Interface Reconstruction Correction for Zones}

To prevent excessive numerical diffusion during the overlay remap, interface reconstruction (IR) is performed using pre-remap volume fractions on the original donor mesh. Per-material mass densities are consequently steepened prior to being remapped to account for sharp subcell interfaces computed by the IR method.

When multiple materials are present, we remap two zonal quantities per material $p$-its volume fraction $\widetilde{f}_{z}^{p}$ and its specific mass density $\widetilde{\rho}_{z}^{p}$-as well as the total mass density

$$
\widetilde{\rho}_{z}=\sum_{p} \widetilde{f}_{z}^{p} \widetilde{\rho}_{z}^{p}
$$

The IR algorithm spatially organizes the location of material volumes within a zone. As a result, density profiles within mixed-material zones are steepened. The total zone density is material-independent and is not steepened by IR. Consequently, the post-remap total zone density $\rho_{z}$ may no longer equal the sum of the post-remap material densities, denoted here by $\rho_{z}^{s}$ :

$$
\rho_{z}^{s}=\sum_{p} f_{z}^{p} \rho_{z}^{p}
$$

This discrepancy typically occurs in target zones intersected by a material interface (see fig. 3). 
We now have two (possibly unequal) values for the post-remap total zone density. Which one does the reconstruction algorithm use to compute subzonal densities? The clear choice is $\rho_{z}^{s}$ : its value has been steepened by the IR step and is thus consistent with all post-remap volume fraction data.

Why then do we bother remapping the total zone density independent of the individual material densities? The reason is that it allows us to quantify the change in mass per zone resulting from IR. Let $\Delta m_{z}^{I R}$ denote the zone mass correction due to IR, defined by

$$
\Delta m_{z}^{I R}=\left(\rho_{z}^{s}-\rho_{z}\right) V_{z}
$$

The utility of eq. (11) is described in the section 4.1.2.
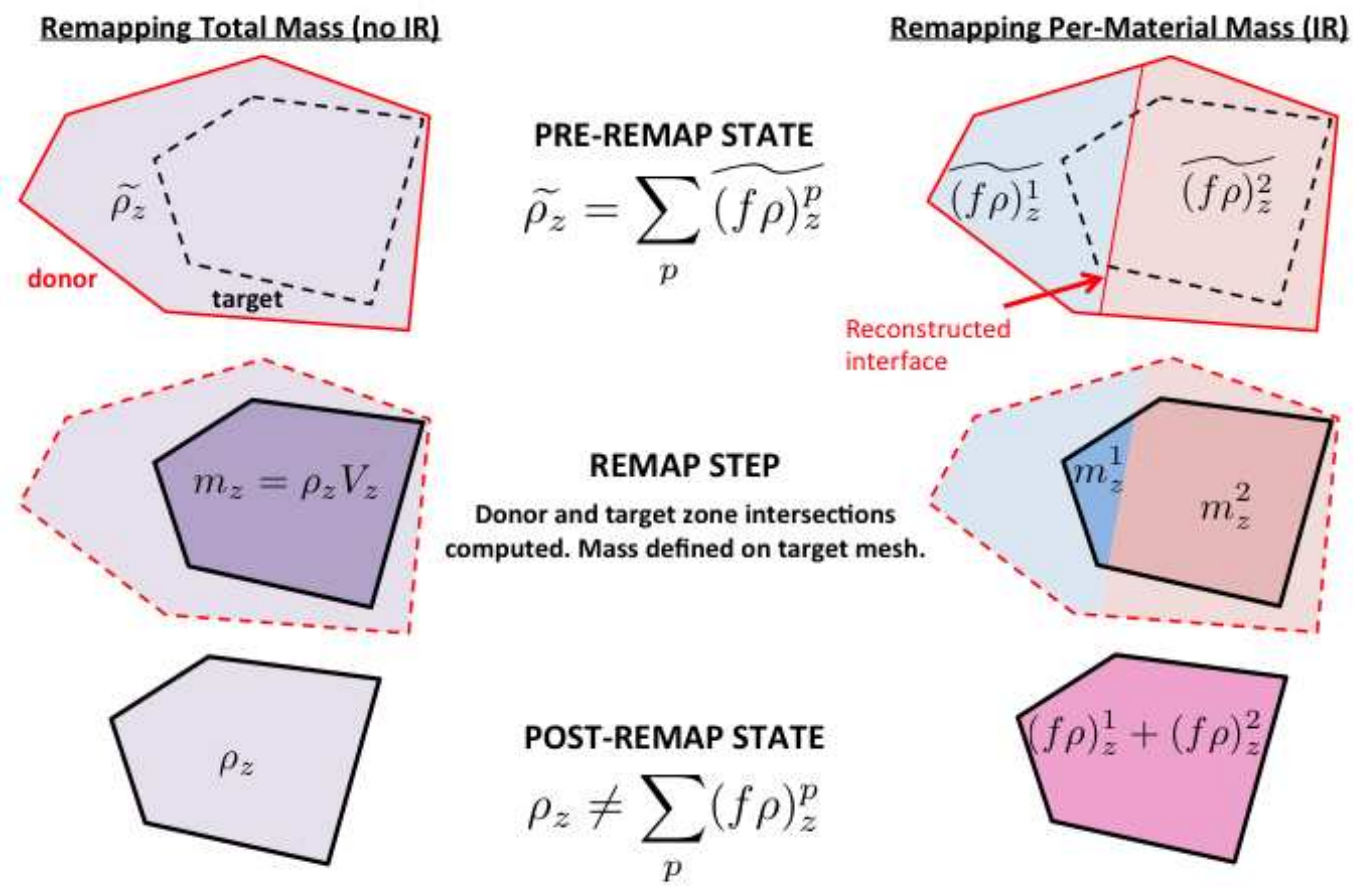

Figure 3: Cartoon comparing the overlay remap of total zone density and individual material densities with interface reconstruction. Front steepening due to interface reconstruction leads to a different total zone density after the remap. 


\subsubsection{Interface Reconstruction Correction for Nodes}

The multimaterial mass overlay summarized in eq. (9) only remaps the total nodal mass density. Because IR is not computed on dual mesh elements, the post-remap nodal density has not been steepened near material interfaces and is likely inconsistent with

$$
\rho_{z}^{s}=\rho_{z}+\Delta m_{z}^{I R} / V_{z} .
$$

The goal of this step is to take the IR mass corrections eq. (11) computed at zones and compute a consistent mass correction at neighboring nodes.

The IR mass correction at node $n$ is defined by a weighted sum of zone mass corrections around $n$ :

$$
\Delta m_{n}^{I R}=\sum_{c(n)} w_{c} \Delta m_{z_{c}}^{I R},
$$

where $w_{c}$ is a nonnegative weight computed at each subzone. Weights are computed so that total nodal mass is conserved. In particular, a sufficient condition for

$$
\sum_{n} \Delta m_{n}^{I R}=0
$$

is the following constraint on the weights:

$$
\sum_{c(z)} w_{c}=1
$$

for each zone in the domain. For this paper, we use a mass-weighted value for $w_{c}$ :

$$
w_{c}=\frac{\rho_{n_{c}} V_{c}}{\bar{m}_{z_{c}}},
$$

where the average mass $\bar{m}_{z}$ per zone is given by

$$
\bar{m}_{z}=\sum_{c(z)} \rho_{n_{c}} V_{c} .
$$

This trivially satisfies eq. (13) and thus conserves total node mass.

Following the notation in eq. (10), we write the corrected node densities

$$
\rho_{n}^{s}=\rho_{n}+\Delta m_{n} / V_{n}=\rho_{n}\left(1+\sum_{c(n)} \frac{V_{c}}{V_{n}} \frac{\Delta m_{z_{c}}^{I R}}{\bar{m}_{z_{c}}}\right) .
$$


Remark: Different expressions for weights $w_{c}$ may be selected that conserve total node mass. In particular, we have experimented with volume-derived weights $w_{c}=V_{c} / V_{z_{c}}$ as well as more complicated expressions involving direct optimization of the corrected node densities $\rho_{n}^{s}$. We found that positivity of $\rho_{n}^{s}$ is not guaranteed for these alternatives if large jumps in specific mass density exist across an interface. The mass-derived weights eq. (14) are guaranteed to preserve positivity because the correction is equivalent to a multiplicative rescaling of the original post-remap value, as implied by the last expression in eq. (16).

\subsubsection{Nodal Density Repair}

It was observed in [23] that the subzonal reconstruction procedure is sensitive to non-monotonicities among the post-remap nodal and zonal densities. For instance, if $\rho_{n}$ is significantly outside the bounds of $\rho_{z}$ for neighboring zones $z(n)$, then the resulting subzonal reconstruction can be non-monotonic. Negative subzonal densities can also occur if the post-remap data has steep density discontinuities.

To alleviate this sensitivity, we employ a nodal density repair procedure similar to the one outlined in [16] following the IR correction step. For node $n$ having corrected density $\rho_{n}^{s}$, we define its density bounds

$$
\rho_{n}^{\min }=\min _{z(n)}(1-\varepsilon) \rho_{z}^{s} \quad \text { and } \quad \rho_{n}^{\max }=\max _{z(n)}(1+\varepsilon) \rho_{z}^{s}
$$

where $0 \leq \varepsilon \ll 1$ is a small number to account for round-off errors. Each node is categorized as being above, below, or within the bounds $\left[\rho_{n}^{\min }, \rho_{n}^{\max }\right]$, and a discrete mass change $\Delta m_{n}^{\text {repair }}$ is computed in attempt to bring each node within its local bounds.

We first define the primary neighborhood of node $n$ as the set of nodes $\mathcal{N}(n)$ that connect to $n$ by an edge. Let $\widehat{\mathcal{N}}(n) \subseteq \mathcal{N}(n)$ denote the primary node neighbors of $n$ that are also within their density bounds. For brevity in describing the algorithm, we only outline the case of a node being above its bounds; the below-bounds case is analogous. Interested readers are encouraged to consult [16] for a more thorough description.

If $\rho_{n}^{s}>\rho_{n}^{\max }$, then mass must be taken from $n$ and distributed proportionally among $\widehat{\mathcal{N}}(n)$ so that all nodes are within bounds. The mass overage at node $n$ is

$$
m_{n}^{\text {over }}=\left(\rho_{n}^{s}-\rho_{n}^{\max }\right) V_{n}
$$


A "mass budget" is computed for all nodes in $\widehat{\mathcal{N}}(n)$ to verify if $m_{n}^{\text {over }}$ can safely be absorbed by them. We define

$$
m_{n}^{\text {budget }}=\sum_{\hat{n} \in \widehat{\mathcal{N}}(n)}\left(\rho_{\hat{n}}^{\max }-\rho_{\hat{n}}^{s}\right) V_{\hat{n}},
$$

And compute the safe mass change for the repair step by

$$
\Delta m_{n}^{\text {repair }}=-\min \left\{m_{n}^{\text {over }}, m_{n}^{\text {budget }}\right\} .
$$

Mass changes for the neighbors of $n$ are computed

$$
\Delta m_{\hat{n}}=\left(\frac{\Delta m_{n}^{\text {repair }}}{m_{n}^{\text {budget }}}\right)\left(\rho_{\hat{n}}^{\text {max }}-\rho_{\hat{n}}^{s}\right) V_{\hat{n}},
$$

where $\hat{n}$ indexes the nodes in $\widehat{\mathcal{N}}(n)$. The procedure is similar if a node is below bounds. Mass from neighboring nodes is donated to bring it into bounds.

\section{Remarks:}

1. If a node's mass density is above its bounds but contains more mass than its neighbors can absorb (i.e. $m_{n}^{\text {over }}>m_{n}^{\text {budget }}$ ), the mass change is limited to that of $m_{n}^{\text {budget }}$. We do not widen the stencil over which the node may donate mass; it simply remains out of bounds.

2. A node that begins within bounds can potentially donate and absorb mass from an arbitrary number of neighbors that are not within bounds. The total mass change for this node is the sum of all such corrections eq. (21). Because mass budgets eq. (19) are computed on a per-node basis, it is not guaranteed that the updated density for this node, given by

$$
\rho_{n}^{s}+\Delta m_{n}^{\text {repair }} / V_{n},
$$

is still within bounds. We get around this complication by iterating the repair procedure. More than two iterations of the repair procedure are seldom needed in practice.

3. Although nodal density repair is non-physical in nature, we consider it a necessary step to remove spurious extrema and improve remap accuracy. Evidence for the accuracy improvement is presented in section 5.1 .3 . 
4. Repair corrects spurious extrema after they have occurred through the overlay remap. Alternate procedures may be devised to correct the remap stage and prevent the extrema in the first place. This is a good direction for future work. One possibility is to use intersection information from the overlay to obtain mass fluxes on the target mesh, as demonstrated in [11]. A flux correction step may be employed to preserve monotonicity. The optimization procedure outlined in [17] would be a good first step.

\subsubsection{Correcting Additional Remapped Nodal Quantities}

The procedure we have outlined in the previous sections corrects the postremap nodal density in a manner that improves consistency with respect to remapped zone data and reduces sensitivities of the reconstruction algorithm to non-monotonic data. If no other quantities beyond mass density need remapping, then the preprocessing stage is complete. If, on the other hand, we wish to remap additional nodal quantities that depend on nodal mass, then we must now correct their values to account for the previous steps. This is certainly true in the case of remapping staggered hydrodynamics data due to the fluid momentum.

The discrete fluid momentum density $\rho_{n} \mathbf{u}_{n}$ is conserved per nodal volume and depends on the fluid velocity vector $\mathbf{u}_{n}$ and the nodal mass density. A conservative overlay remaps the nodal density and momentum density

$$
(\widetilde{\rho}, \widetilde{\rho \mathbf{u}})_{n} \longrightarrow(\rho, \rho \mathbf{u})_{n}
$$

The post-remap velocity is then recovered

$$
\mathbf{u}_{n}=(\rho \mathbf{u})_{n} / \rho_{n}
$$

If a correction is made to the post-remap nodal density, then the post-remap momentum density becomes inconsistent, and the recovered velocity is in error.

We present a method for correcting the momentum in a manner that (i) is conservative, (ii) preserves exactly the direction of the post-remap velocity, and (iii) attempts to preserve the relative velocity magnitude as best as possible. We express the details of the method in terms of fluid momentum, but the ideas generalize to any scalar or vector quantity discretized on nodes 
that is conserved with respect to the nodal mass density. Note that in the case of scalar quantities (ii) is obtained automatically.

We begin by defining the fluid momentum

$$
\mathbf{p}_{n}=m_{n} \mathbf{u}_{n}
$$

and decomposing it into its magnitude and direction

$$
\mathbf{p}_{n}=p_{n} \hat{d}_{n}=m_{n} u_{n} \hat{d}_{n}
$$

where $p_{n}, u_{n}$, and $\hat{d}_{n}$ represent the momentum magnitude, velocity magnitude, and direction vector respectively. Given the total change to nodal mass

$$
\Delta m_{n}=\Delta m_{n}^{I R}+\Delta m_{n}^{\text {repair }}
$$

and the final corrected nodal mass

$$
m_{n}^{s}=m_{n}+\Delta m_{n}
$$

we compute corrections $\Delta p_{n}$ that satisfy the following constrained optimization problem:

$$
\begin{array}{ll}
\text { minimize: } & \frac{1}{2} \sum_{n}\left(u_{n}^{2}+\varepsilon^{2}\right)^{-1}\left(\frac{p_{n}+\Delta p_{n}}{m_{n}+\Delta m_{n}}-\frac{p_{n}}{m_{n}}\right)^{2} \\
\text { subject to: } & \sum_{n} \Delta p_{n} \hat{d}_{n}=0
\end{array}
$$

where again $\varepsilon$ is a small number to avoid division by zero. The term $\left(u_{n}^{2}+\right.$ $\left.\varepsilon^{2}\right)$ normalizes the variation to prevent large velocities form dominating the correction term. The objective of eq. (26) is to solve for globally conservative corrections to $\mathbf{p}_{n}$ so that the relative difference in the corrected and postremap velocities is as small as possible under $L_{2}$ norm.

Similar to the problem of subzonal reconstruction, eq. (26) leads to an under-constrained system that can be solved by the method of Lagrange multipliers. This system can be solved directly for $\Delta p_{n}$ :

$$
\Delta p_{n}=u_{n} \Delta m_{n}-\left(m_{n}^{s} u_{n}\right)^{2}\left(\mathbf{z} \cdot \hat{d}_{n}\right)
$$


where $\mathbf{z}$ is the vector of Lagrange multipliers of length equal to $D$, the dimension of the problem. The multipliers satisfy the symmetric linear system:

$$
\begin{gathered}
\sum_{j=1}^{D} a_{i, j} z_{j}=b_{i} \\
a_{i, j}=\sum_{n}\left(m_{n}^{s} u_{n}\right)^{2} \hat{d}_{n}^{(i)} \hat{d}_{n}^{(j)} \\
b_{i}=\sum_{n} u_{n} \Delta m_{n} \hat{d}_{n}^{(i)}
\end{gathered}
$$

where $1 \leq i, j \leq D$ and $\hat{d}_{n}^{(i)}$ denotes the $i$-th component of the direction vector. The corrected velocity may be written

$$
\mathbf{u}_{n}^{s}=\mathbf{u}_{n}\left(1-m_{n}^{s} u_{n}\left(\mathbf{z} \cdot \hat{d}_{n}\right)\right)
$$

Remarks:

1. If velocity is constant everywhere, the solution to system eq. (28) eq. (30) is $\mathbf{z}=0$. Therefore, the corrected velocity equals the postremap velocity, and conservation is ensured automatically.

2. If the post-remap velocity is zero at a node, then its corrected velocity will also be zero. That is, the momentum correction does not produce spurious velocity signals.

3. The optimization problem in eq. (26) is globally coupled, because we sum over all nodes for both the objective function and the conservation constraint. An improvement to this algorithm would involve limiting the sums over those nodes having $\Delta m_{n} \neq 0$. This would localize the impact of the momentum correction to discontinuous regions of the problem (i.e. shocks and material interfaces).

\subsection{Stage 2: Subzonal Reconstruction}

The total subzonal mass is reconstructed using the procedure outlined in [23] and denoted by the reconstruction operator $\mathcal{R}$. The reconstruction

procedure takes as input the remapped (and corrected) values of total zone and node masses. We write

$$
\rho_{c}=\mathcal{R}\left(m_{z}^{s}, m_{n}^{s}\right)
$$


where the IR corrected zone mass is

$$
m_{z}^{s}=m_{z}+\Delta m_{z}^{I R}
$$

and the corrected node mass is

$$
m_{n}^{s}=m_{n}+\Delta m_{n}^{I R}+\Delta m_{n}^{r e p a i r}
$$

Note that in the single-material case, we have that $\Delta m_{z}^{I R}, \Delta m_{n}^{I R}$, and $\Delta m_{n}^{\text {repair }}$ are all zero and we recover the original procedure.

To summarize [23], subzonal densities are reconstructed by solving the following constrained optimization problem:

$$
\begin{gathered}
\rho_{c}=\underset{\rho_{c}}{\operatorname{argmin}} \sum_{c}\left(\frac{\rho_{c}-\mu_{c}}{\mu_{c}}\right)^{2} \\
\text { subject to: }\left\{\begin{array}{l}
\sum_{c(z)} \rho_{c} V_{c}=m_{z}^{s}, \quad \forall z \\
\sum_{c(n)} \rho_{c} V_{c}=m_{n}^{s}, \quad \forall n
\end{array}\right.
\end{gathered}
$$

where $V_{c}$ are the known subzonal volumes of the target mesh, and $\mu_{c}$ is a reference subzonal density. Our findings in [23] suggest that a harmonic average of the remapped zone and node densities offers the optimal combination of accuracy and stability for the optimization problem. This is particularly true when steep discontinuities in density exist in the state. Therefore we use the harmonic reference value

$$
\mu_{c}=\frac{2 \rho_{z_{c}}^{s} \rho_{n_{c}}^{s}}{\rho_{z_{c}}^{s}+\rho_{n_{c}}^{s}},
$$

where $\rho_{z}^{s}=m_{z}^{s} / V_{z}$ and $\rho_{n}^{s}=m_{n}^{s} / V_{n}$.

We compute eq. (34)-eq. (35) by solving a sparse, global linear system. Many efficient software packages exist to solve the resulting system in both serial and parallel. We use the algebraic multi-grid (AMG) preconditioned GMRES solver available in the software package Hypre [7]. 
Remark: The sparse, global matrix arising from eq. (34) - eq. (35) is singular due to the fact that we are solving a least-squares problem with no unique solution. First, it is worth pointing out that a solution to the reconstruction problem is guaranteed to exist: one such solution being the result of a direct subcell remap. Second, because the matrix is singular, the AMG preconditioner must be modified to account for this: namely, we turn off direct matrix inversion for the coarsest multi-grid level solve. This is enough to avoid the codimension-1 null space of the matrix.

\subsection{Stage 3: Assignment}

At this stage, we have a total subzonal mass density that is conservative with respect to zonal and nodal representations of interface-reconstructed, post-remap mass. What remains is to assign specific mass densities per material to each subzone.

Recall that our discrete multimaterial model assigns material volume concentrations to zone elements; subzonal elements are assumed to have the same material concentrations as the zone that contains them. We therefore assert that the ratio of specific mass density to total mass density within a zone is equal to the same ratio inside each of its subzones:

$$
\rho_{c}^{p}=\left(\frac{\rho_{z_{c}}^{p}}{\rho_{z_{c}}}\right) \rho_{c}
$$

and $f_{c}^{p}=f_{z_{c}}^{p}$ as before.

\subsection{Summary}

For convenience and clarity, we summarize the multimaterial reconstruction algorithm here. We use as an example the remapping of state variables for ALE hydrodynamics.

The initial conditions of the algorithm consists of the following staggered post-remap state. Material-independent data: total zonal mass density $\rho_{z}$, total nodal mass density $\rho_{n}$, total momentum density $(\rho \mathbf{u})_{n}$, and total kinetic energy density $K_{n}=\frac{1}{2} \rho\left\|\mathbf{u}_{n}\right\|^{2}$. Also included is material-dependent zone-centered data: volume fractions $f_{z}^{p}$, specific mass densities $\rho_{z}^{p}$, and internal energy densities $\epsilon_{z}^{p}$. The algorithm proceeds:

\section{Preprocess the Post-Remap State}


- Compute the zone mass correction $\Delta m_{z}^{I R}$ due to interface reconstruction (IR) using eq. (10) and eq. (11).

- Scatter $\Delta m_{z}^{I R}$ to neighboring nodes using eq. (12) - eq. (15) to compute the IR-corrected total node density eq. (16).

- Restore monotonicity to the total node density using the conservative repair procedure described in eq. (17) - eq. (21).

- Apply a conservative correction to the post-remap momentum that is consistent with the corrections made to total nodal density due to IR and repair. The correction we derive is summarized in eq. (27) and eq. (31).

\section{Apply Reconstruction Algorithm}

- Compute the collection of total subzonal densities which satisfy the optimization problem eq. (34) subject to conservation constraints eq. (35).

\section{Assign Multimaterial Subzonal Densities}

- Compute material-dependent subzonal mass densities $\rho_{c}^{p}$ using the total subzonal densities computed in Stage 2 and eq. (37).

- The form of eq. (37) assumes that the per-material mass fraction in each subzone equals the per-material mass fraction of the zone containing it. This is consistent with a discrete multimaterial model centered on zones.

The reconstructed material-dependent subzonal densities are conservative with respect to total zone mass, total node mass, and per-material mass. Conserved nodal quantities depending on nodal density (for instance, momentum) are also corrected in a conservative manner. Overall, the procedure outlined above is fully conservative in mass and momentum for multimaterial staggered, compatible hydrodynamics data. Conservation of energy is similarly maintained using a kinetic energy fix-up procedure like the one mentioned in [17], which is why we have included the kinetic energy as one of our remapped variables.

\section{Numerical Results}

We validate our new procedure using a collection of multimaterial test problems. First is a set of pure remapping tests with no discrete physics; second is a set of multimaterial ALE hydrodynamics tests. 
As with the single-material reconstruction algorithm in [23], the new multimaterial extension algorithm has been implemented in LLNL's production multi-physics code KULL [19]. All intersection remap operations are performed using the geometrical overlay package Overlink [6].

Prior to development of the subzonal reconstruction algorithm, KULL flattened subzonal densities across a zone following an overlay remap. All subzonal densities within a zone were set equal to the post-remap zone density. This method is insufficient for hydrodynamics, because linear momentum is not conserved following this procedure. The benefits of reconstructing subzonal densities over flattening them were demonstrated extensively in [23] for single-material hydro problems. No comparisons to the non-conservative flattening algorithm are supplied here.

For the pure remapping tests we determine solution accuracy using the $L_{1}$ error

$$
E_{L_{1}}=\frac{1}{M} \sum_{i=1}^{M}\left|a_{i}-b_{i}\right|,
$$

where $\left\{a_{i}\right\}_{i}$ and $\left\{b_{i}\right\}_{i}$ are length- $M$ sequences representing the discrete postremap state and the pre-remap state (i.e. exact solution) respectively.

\subsection{Pure Remapping Tests}

In this section we examine several 2D pure remapping tests performed in unit square meshes. We consider various combinations of smoothed, discontinuous, and linear profiles for density and volume fractions to test the reconstruction of material-dependent subzonal density. We define 1D profiles aligned arbitrarily in 2D space thusly: let $\xi=\widehat{\mathbf{n}} \cdot(\mathbf{x}-\mathbf{c})$ define a $1 \mathrm{D}$ coordi-

nate system with direction $\widehat{\mathbf{n}}$ passing through $\mathbf{c}$. The line $\xi=0$ corresponds to a line having normal direction $\widehat{\mathbf{n}}$ dividing the unit square into two regions. We define three different distribution functions: a discontinuous profile

$$
h_{d}(\mathbf{x})=h_{d}(\xi(\mathbf{x}))= \begin{cases}0, & \xi \leq 0 \\ 1, & \xi>0\end{cases}
$$


a smoothed continuous profile with smoothing scale $\epsilon$

$$
h_{\epsilon}(\mathbf{x})=h_{\epsilon}(\xi(\mathbf{x}))= \begin{cases}0 & , \quad \xi \leq-\epsilon \\ \frac{1}{2}+\frac{\xi}{\epsilon}+\frac{\xi^{2}}{2 \epsilon^{2}} & , \quad-\epsilon<\xi \leq 0 \\ \frac{1}{2}+\frac{\xi}{\epsilon}-\frac{\xi^{2}}{2 \epsilon^{2}} & , \quad 0<\xi \leq \epsilon \\ 1 & , \quad \xi>\epsilon\end{cases}
$$

and a radial discontinuous profile based on two concentric circles

$$
h_{r}(\mathbf{x})= \begin{cases}1, & r_{\text {inner }}<\|\mathbf{x}-\mathbf{c}\|<r_{\text {outer }} \\ 0, & \text { else }\end{cases}
$$

For all cases we use $\widehat{\mathbf{n}}=(4 / 5,3 / 5), \mathbf{c}=(0.5,0.5), r_{\text {inner }}=0.25$, and $r_{\text {outer }}=$ 0.45 .

Distributions eq. (39) - eq. (41) define profiles for the initial total density and volume fractions:

$$
\rho(\mathbf{x})=\rho_{L}+\left(\rho_{H}-\rho_{L}\right) h(\mathbf{x})
$$

and

$$
f^{1}(\mathbf{x})=h(\mathbf{x}) \quad f^{2}(\mathbf{x})=1-f^{1}(\mathbf{x}),
$$

where $h$ can be $h_{d}, h_{\epsilon}$, or $h_{r}$. In addition, we define a linear total density profile by

$$
\rho(\mathbf{x})=\rho_{0}+\nabla \rho \cdot \mathbf{x}
$$

In each case, we use $\rho_{H}=1, \rho_{L}=0.01, \rho_{0}=1$, and $\nabla \rho=(1,5)$. We experiment with different smoothing scales $\epsilon$ in our testing.

Discrete subzonal density is computed by simply evaluating the continuous initial density profile: $\rho_{c}=\rho\left(\mathbf{x}_{c}\right)$, where $\mathbf{x}_{c}$ is the barycenter of subzone $c$. Discrete volume fractions are defined in a consistent manner: a subzonal volume fraction is computed by evaluating the continuous distribution then summed with volume weights to produce a zonal volume fraction:

$$
f_{z}^{1}=\frac{1}{V_{z}} \sum_{c(z)} f^{1}\left(\mathbf{x}_{c}\right) V_{c} \quad f_{z}^{2}=1-f_{z}^{1},
$$

where the superscripts ${ }^{1}$ and ${ }^{2}$ indicate material numbers. 
Finally, we have some freedom in defining the discrete specific densities $\rho^{1}$ and $\rho^{2}$ at each subzone. To further test the algorithm, we initialize them to have an order of magnitude discrepancy: $\rho^{2}=0.1 \rho^{1}$. Thus,

$$
\rho_{c}^{1}=\frac{\rho_{c}}{f_{z_{c}}+0.1\left(1-f_{z_{c}}\right)} \quad \text { and } \quad \rho_{c}^{2}=\frac{0.1 \rho_{c}}{f_{z_{c}}+0.1\left(1-f_{z_{c}}\right)} .
$$

Figure 4 illustrates what a few of the volume fraction and density profile combinations look like in 1D.
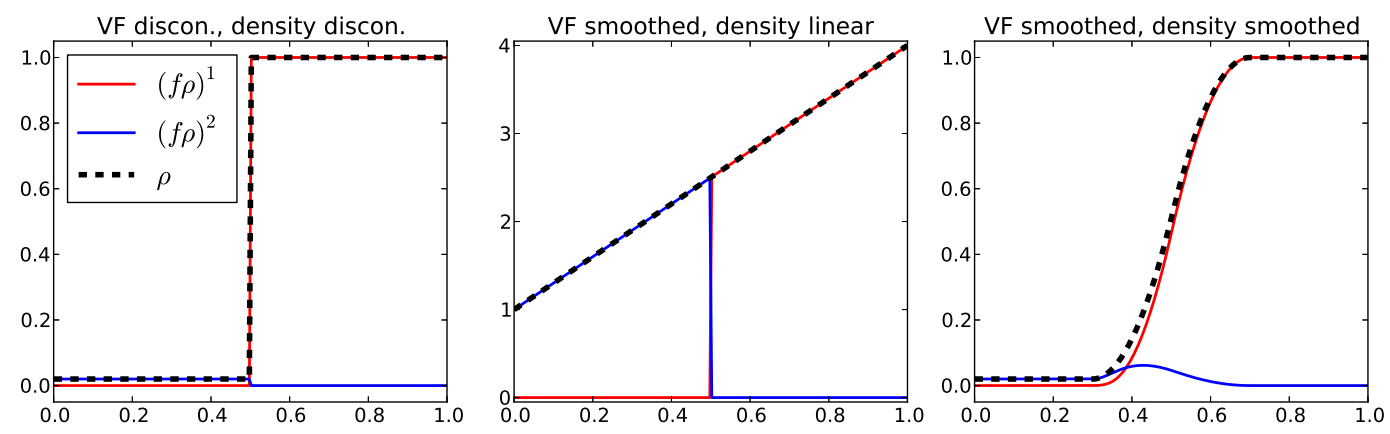

Figure 4: Example 1D profiles of per-material densities and total density. Volume fraction interfaces are discontinuous (left) and smoothed (center and right). Total density is discontinuous (left), linear (center), and smoothed (right).

\subsubsection{2-Material Null Problem}

We start with an initial unit lattice mesh with $N \times N$ zone elements, remap a discrete multimaterial density state onto the same mesh, and reconstruct the material-dependent subzonal data. The null remap ensures that post-remap quantities on each zone and node are exact to within computer precision. This allows us to test how well the new algorithm reconstructs material-dependent subzonal information from exact density data on zones and nodes.

We compute $L_{1}$ convergence in subzonal density for both the total and the per-material components for different combinations of initial density and volume fraction profiles. Grid convergence is established for $N \in\{16,32,64,128,256\}$. Figure 5 illustrates $L_{1}$ convergence in total subzonal density. The rates of convergence depend on the smoothness in the initial density field, as expected. Discontinuous density profiles converge at first order; linear and 
smoothed-discontinuous profiles converge at second order. A constant smoothing scale of $\epsilon=0.1$ was used in this calculation.

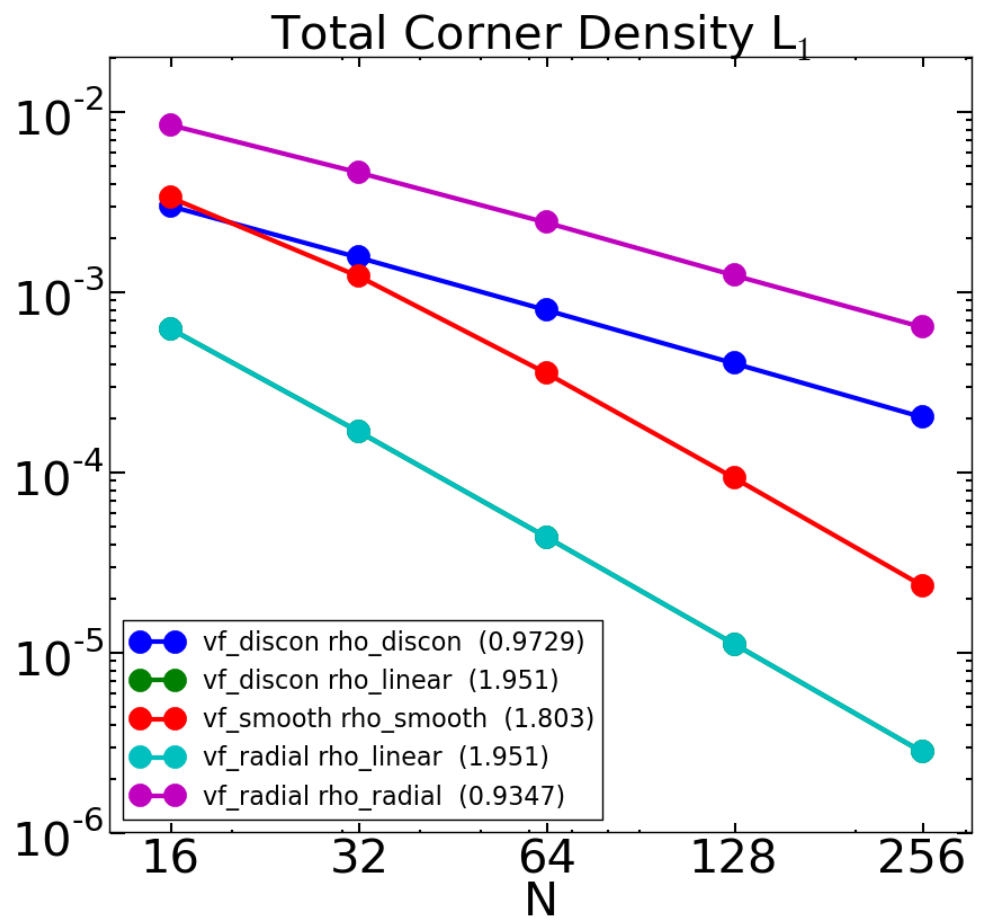

Figure 5: Two-material null problem: $L_{1}$ convergence in total reconstructed subzonal density. Note that "vf_discon rho_linear" and "vf_radial rho_linear" coincide.

Figure 6 gives $L_{1}$ convergence in the two material-dependent subzonal densities. Convergence rates are consistent with total density. Absolute error differs due to differences in specific density. Altogether, the new algorithm does a good job reconstructing material-dependent subzonal mass distributions from exact representations of zonal and nodal mass density.

\subsubsection{Reconstructing a Material Interface}

Next we test the multimaterial reconstruction algorithm using approximate remap data. We map material interface data on an $N \times N$ lattice grid onto three different grids: a random Voronoi tessellation, a lattice grid with high-aspect-ratio elements, and an optimized centroidal Voronoi tessellation. 

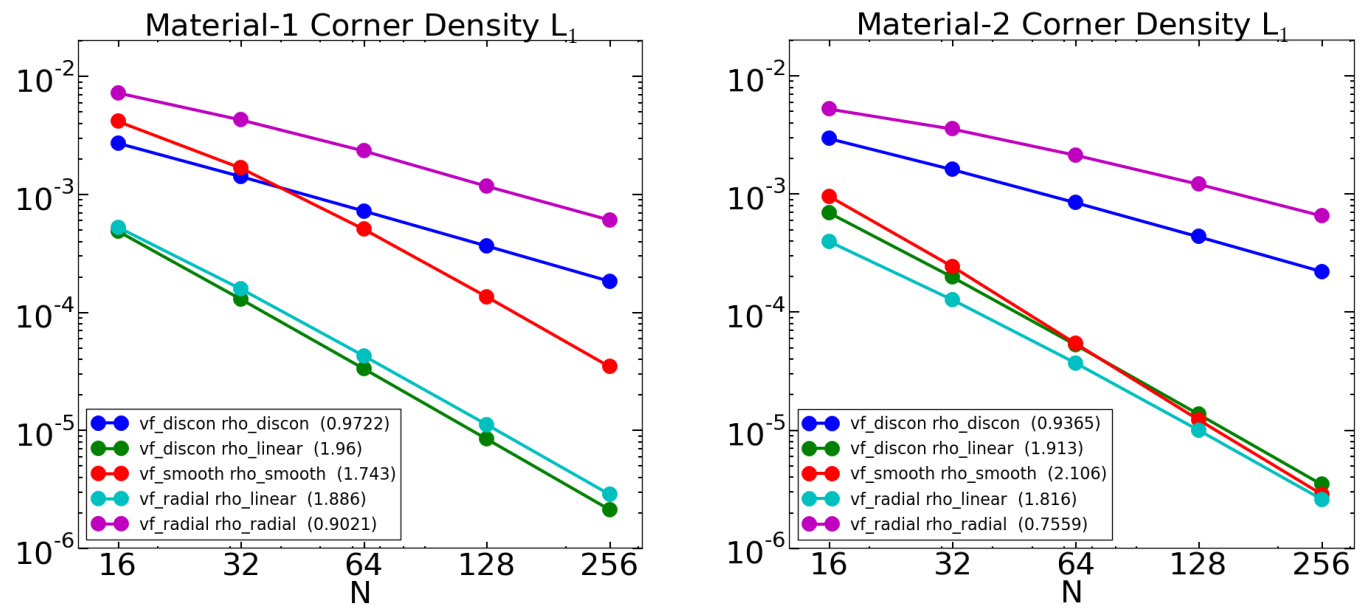

Figure 6: Two-material null problem: $L_{1}$ convergence in reconstructed material-dependent subzonal densities

The high-aspect-ratio case is obtained by taking the initial lattice grid and reducing the number of zones in the vertical direction by a factor of 4 while increasing the number in the horizontal direction by a factor of 4 . The random Voronoi tessellation is obtained by randomly distributing generation sites inside the unit square. The centroidal Voronoi tessellation is obtained through 100 iterations of Lloyd's algorithm on the random tessellation. Figure 7 illustrates each of the three target meshes for $32 \times 32$ zones along with post-remap volume fraction data from a material discontinuity.

The remapped zone density, node density, and volume fractions are approximate and first-order order accurate under grid refinement. The reconstruction algorithm maintains the same level of accuracy with respect to total subzonal density and material-dependent subzonal density. Error is computed between the reconstructed quantities on the post-remap mesh and an "exact" solution obtained by computing the initial pre-remap profiles on the post-remap mesh.

Figure 8 establishes first-order convergence of the reconstructed materialdependent subzonal densities for a sharp material discontinuity. We initialize total density and volume fractions using eq. (39), eq. (42), and eq. (43) and the settings described in section [5.1. Absolute error is larger for the high-aspect-ratio quads due to the drastically reduced grid resolution in the 

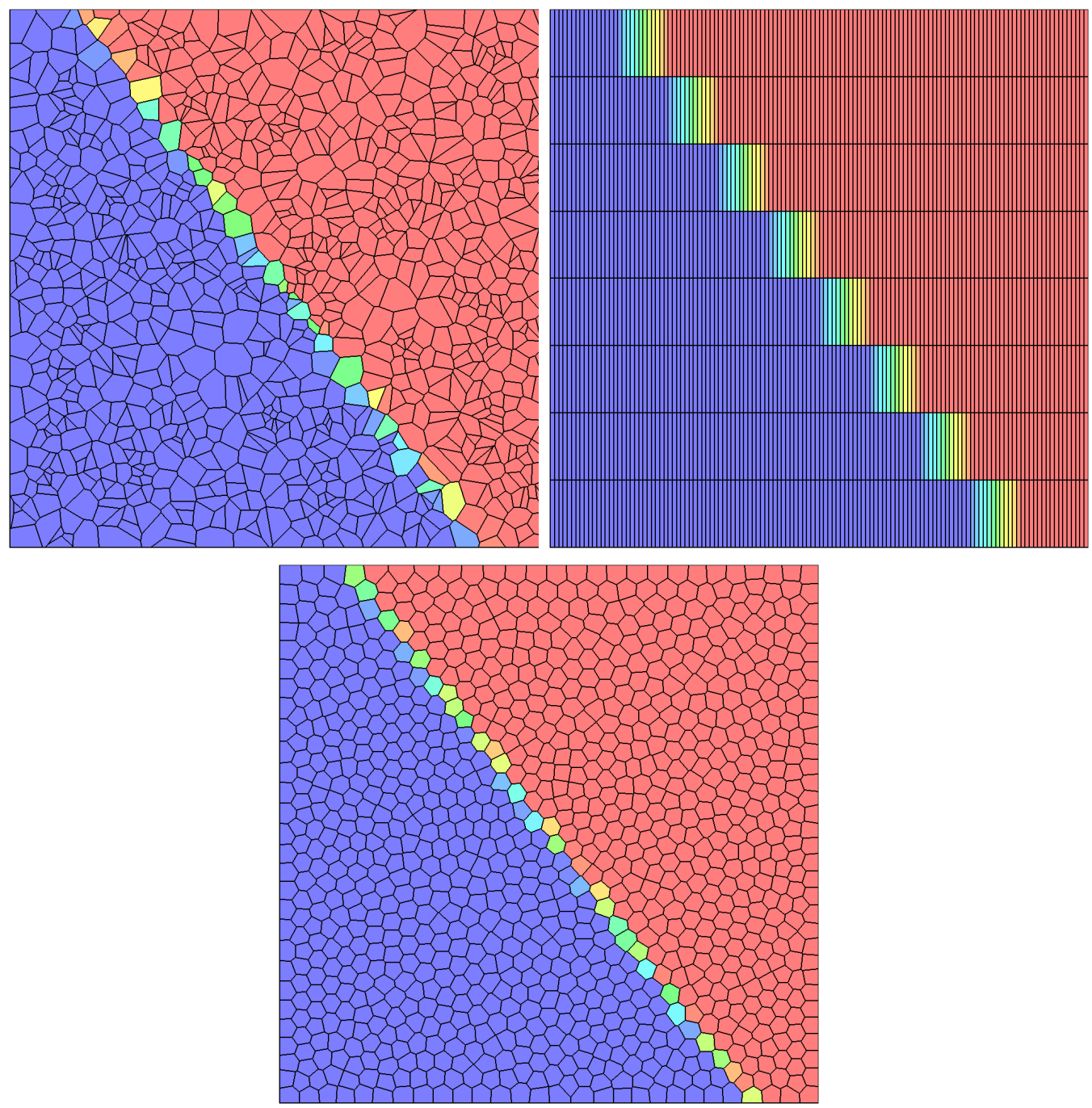

Figure 7: Post-remap volume fraction data from a material discontinuity on a random Voronoi tessellation, a lattice mesh of high-aspect-ratio quads, and a centroidal Voronoi tessellation.

vertical direction. Surprisingly, absolute error is comparable for both the random Voronoi grid and the optimized centroidal Voronoi grid. We expect the centroidal grid to have lower error due to the fact that its subzonal element volumes are approximately equal. Instead we find that the reconstruction algorithm maintains its accuracy even when the post-remap mesh is not as 
well-conditioned.
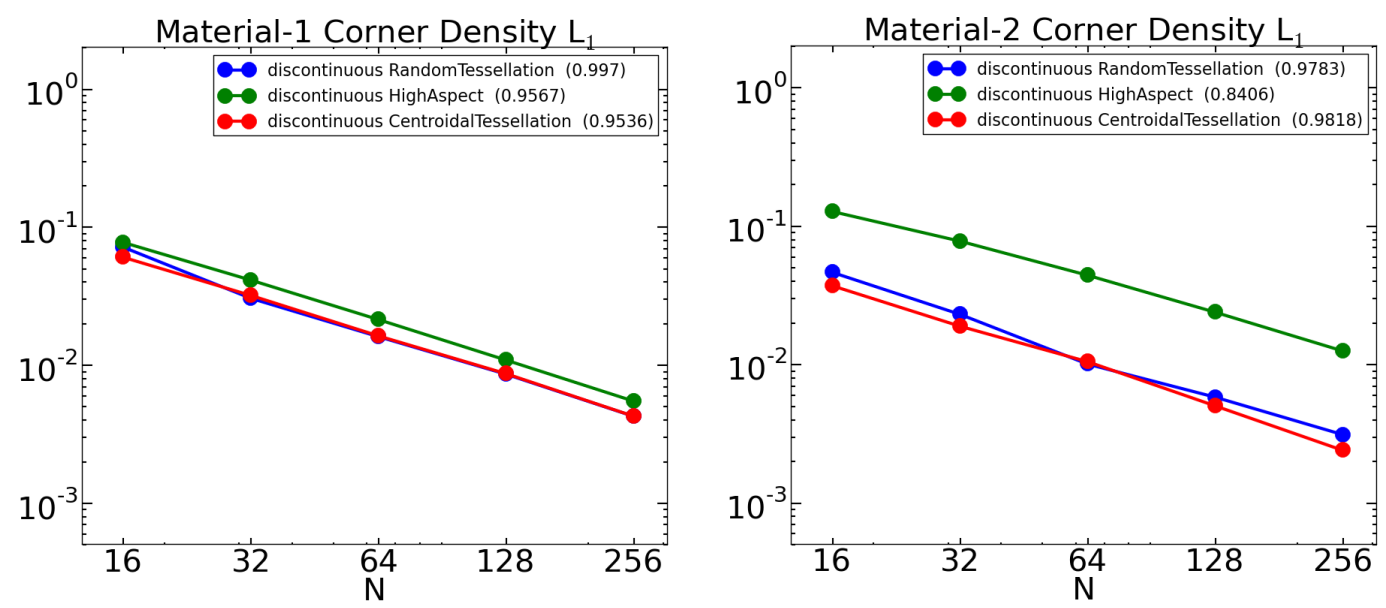

Figure 8: $L_{1}$ convergence rates for the two material discontinuous remap test, with target meshes consisting of a random tessellation, a mesh of high-aspect ratio zones, and a centroidally relaxed Voronoi.

We repeat the test using a smoothed discontinuity. We initialize total density and volume fractions using eq. (40), eq. (42), and eq. (43). The smoothing scale is chosen to place approximately 4 pre-remap grid cells across the interface: $\epsilon=2 / N$. The interface is therefore captured under grid refinement and convergent with respect to interface reconstruction. First order convergence is established, with rates reported in table 1. The Voronoi grids display lower absolute error due to the fact their elements cover the unit square more evenly than the high-aspect-ratio quads do.

\begin{tabular}{c|ccc}
\hline Material & Random tess. & High aspect & Centroidal tess. \\
\hline 1 & 0.875 & 0.903 & 0.885 \\
2 & 0.902 & 0.763 & 0.897 \\
\hline
\end{tabular}

Table 1: $L_{1}$ convergence rates for the two-material smoothed discontinuity remap test.

Finally, we remap the radial shell discontinuity given by eq. (41), eq. (42), and eq. (43). We report the $L_{1}$ convergence results in table 2, First-order 
convergence is again established with absolute error trends comparable to the previous tests.

\begin{tabular}{c|ccc}
\hline Material & Random tess. & High aspect & Centroidal tess. \\
\hline 1 & 0.938 & 0.839 & 0.901 \\
2 & 0.871 & 0.626 & 0.818 \\
\hline
\end{tabular}

Table 2: $L_{1}$ convergence rates for the radial shell discontinuous remap test.

\subsubsection{Multimaterial Cyclic Remap}

We repeat the remapping test from [13] used in [17, 23]. Nodes of the initial mesh are iteratively moved according to a periodic displacement field

$$
\begin{aligned}
& x_{k}\left(x_{0}, y_{0}, \tau\right)=x_{0}+F(\tau) \sin \left(2 \pi \tau x_{0}\right) \sin \left(2 \pi \tau y_{0}\right) \\
& y_{k}\left(x_{0}, y_{0}, \tau\right)=y_{0}+F(\tau) \sin \left(2 \pi \tau x_{0}\right) \sin \left(2 \pi \tau y_{0}\right) \\
& F(\tau)= \begin{cases}0.2 \tau & , \quad \tau \in[0,0.5] \\
0.2(1-\tau) & , \quad \tau \in[0.5,1]\end{cases} \\
& \tau=k / k_{\max }
\end{aligned}
$$

where $k$ marks the displacement iteration, $\left(x_{0}, y_{0}\right)$ is an initial node position, $\left(x_{k}, y_{k}\right)$ denotes the position after $k$ iterations, and $\tau$ indicates a pseudotime period for the displacement field. Mesh nodes return to their original positions after $k_{\max }$ iterations.

We select a difficult initial configuration of materials, densities, and velocity vectors to test the new algorithm. We use a variant of the "ball and jack" material remap problem from [3]. The domain is divided into three regions: a radial shell (the "ball") having density 1 and unit, outward-pointing velocity, a cross-shaped inner region (the "jack") having density 10 and unit, inward-pointing velocity, and a background region having density 0.1 and zero velocity. We initialize the shell to have inner radius $r_{\text {inner }}=0.28125$ and outer radius $r_{\text {outer }}=0.4375$. The cross has length and width dimensions $L=0.375$ and $w=0.125$ and is centered at $(0.5,0.5)$. (See fig. 9.)

Density, volume fraction, and momentum data is overlaid onto the new displaced mesh at every iteration. We measure $L_{1}$ error in total zone density, 

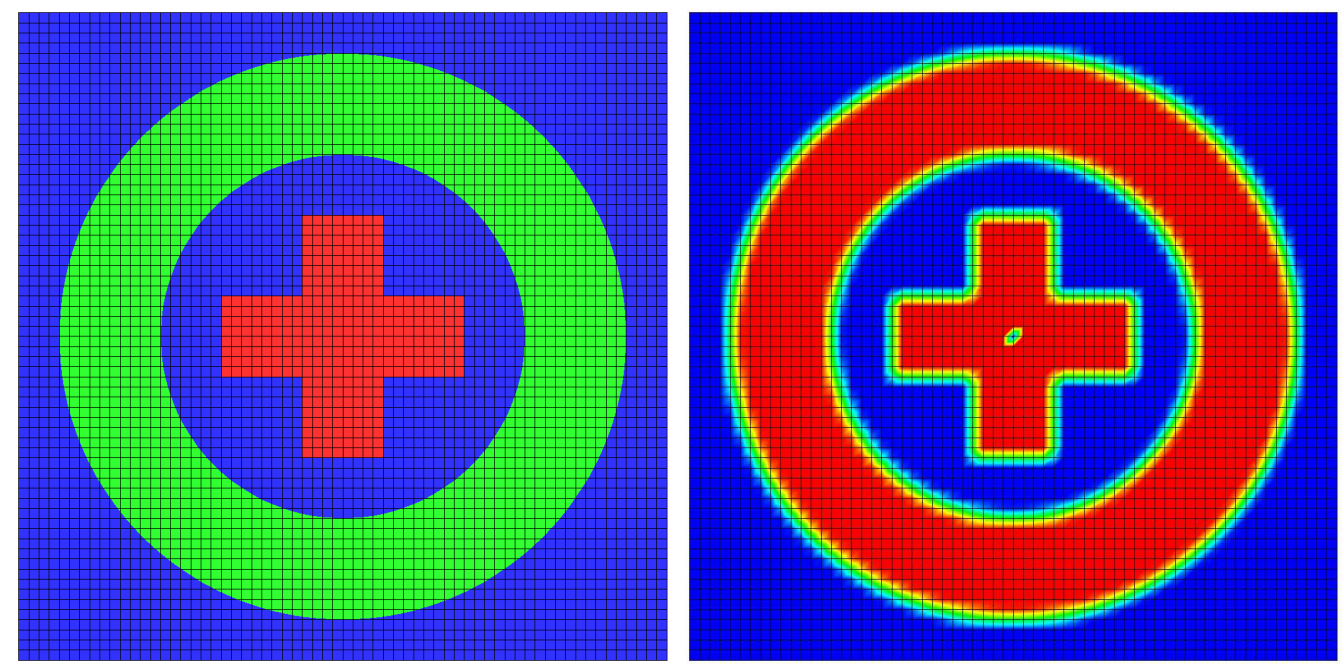

Figure 9: Multimaterial cyclic remap: materials (left) and initial velocity magnitude (right).

total node density, material-dependent subzonal density, and radial velocity. Error is computed at final iteration $k_{\max }=320$ with respect to the initial state. To illustrate the effects of the cyclic remap, we plot the magnitude of the velocity field at maximum mesh displacement and at the final iteration in fig. 10.

The cyclic remap problem is an effective stress test of the new algorithm. It exposes the need for a nodal density repair step to improve convergence of both the post-remap state and the reconstructed subzonal state. Figure 11 gives the convergence trends for total nodal density, radial velocity, and tangential velocity with and without nodal repair. Velocity is not significantly impacted by the repair step, but nodal density certainly is. Grid convergence without repair is sub-linear, with order-of-magnitude larger absolute error than we find when using the repair algorithm. The repaired nodal density converges with the expected first-order rate for this discontinuous problem. In addition, monotonicity in nodal density is preserved when repair is turned on. The minimum and maximum bounds are initially $\left(\rho_{n}^{\min }, \rho_{n}^{\max }\right)=(0.1,10)$ for this problem. At the final iteration, the bounds are $(0.096,10.02)$ with repair turned on and $(0.058,12.03)$ with repair turned off.

Improving the convergence of remapped nodal density ultimately im- 

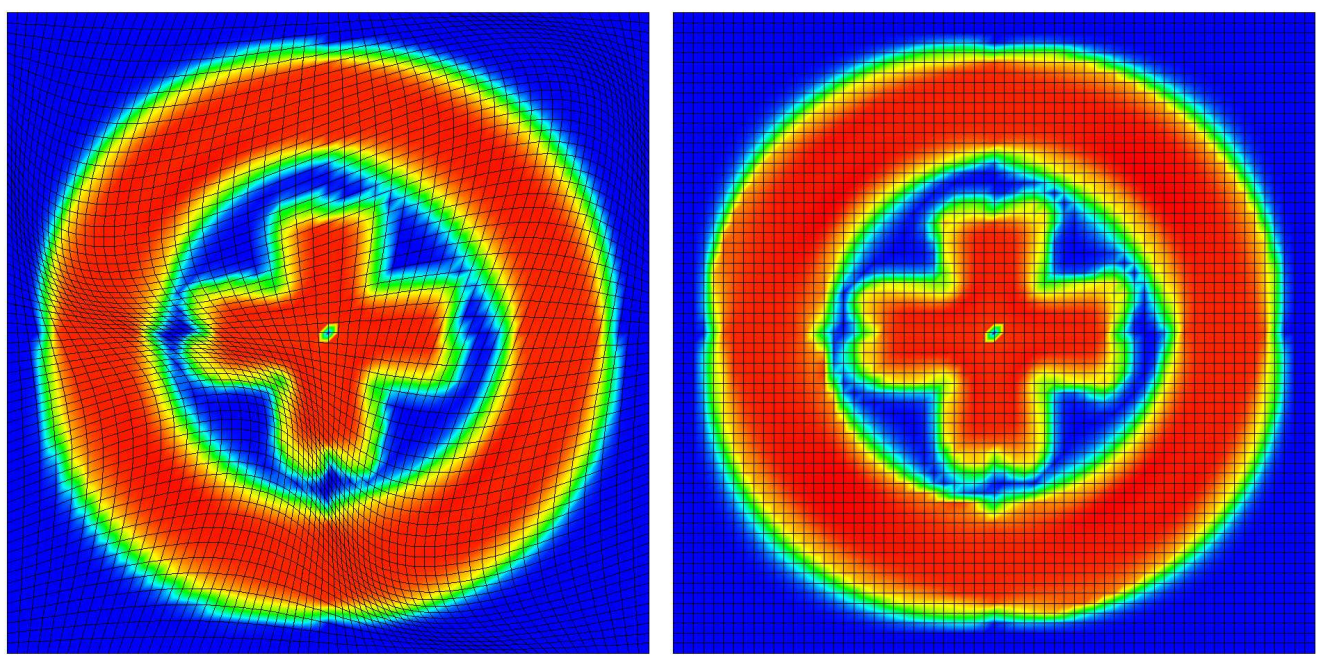

Figure 10: Multimaterial cyclic remap: velocity magnitude at maximum displacement (left) and final iteration (right).
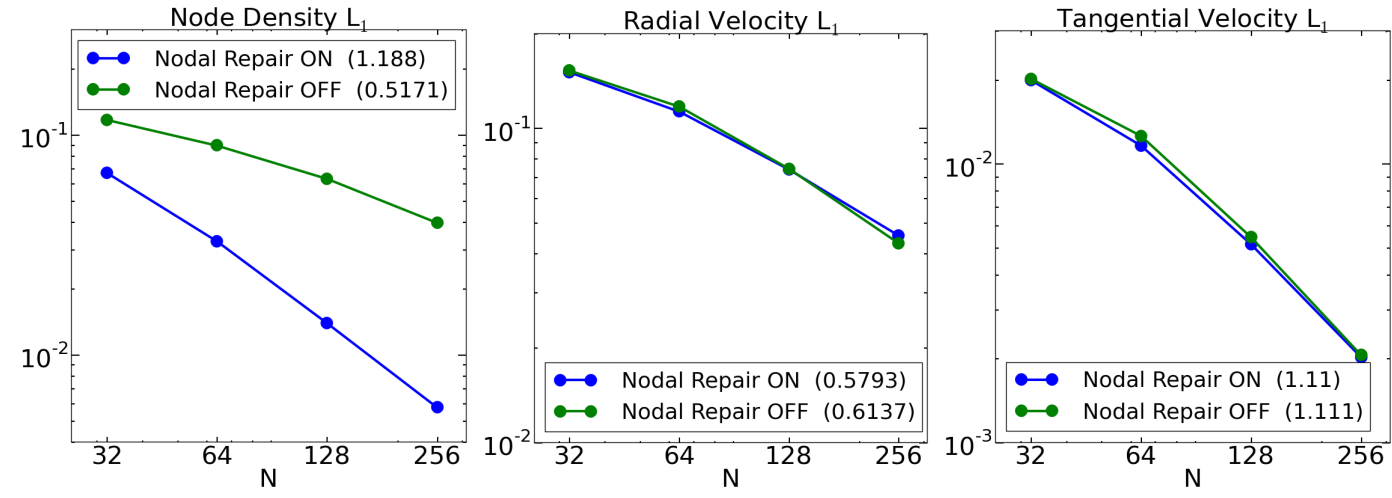

Figure 11: Multimaterial cyclic remap: $L_{1}$ convergence of total nodal density, radial velocity, and tangential velocity with and without nodal repair turned on.

proves convergence of the reconstructed subzonal densities. Table 3 compares the $L_{1}$ error in each material's reconstructed subzonal density with and without repair. Over all, the repair step improves the accuracy of the reconstructed values, with absolute error observed to be $2-5$ times smaller than non-repaired values. The convergence rates for the background, shell, 
and cross materials are computed as $(1.08,0.32,0.69)$ with repair turned on and $(0.20,0.23,0.27)$ with repair turned off.

\begin{tabular}{c|ccc|ccc}
\hline \multirow{2}{*}{$N$} & \multicolumn{3}{|c|}{ Nodal Repair ON } & \multicolumn{3}{c}{ Nodal Repair OFF } \\
& Back. & Shell & Cross & Back. & Shell & Cross \\
\hline 32 & 0.0058 & 0.0194 & 0.0861 & 0.0089 & 0.0576 & 0.1364 \\
64 & 0.0021 & 0.0130 & 0.0482 & 0.0096 & 0.0526 & 0.1215 \\
128 & 0.0011 & 0.0111 & 0.0282 & 0.0085 & 0.0439 & 0.0986 \\
256 & 0.0006 & 0.0098 & 0.0211 & 0.0059 & 0.0357 & 0.0787 \\
\hline
\end{tabular}

Table 3: Multimaterial cyclic remap: $L_{1}$ errors in material-dependent corner densities with and without a monotonicity repair step for post-remap nodal density.

As a final test, we repeat the cyclic remap problem using a non-lattice mesh. Figure 12 illustrates the initial materials on a "honeycomb" mesh composed of approximately $64^{2}$ hexagonal elements. Similarly, fig. 13 gives the velocity magnitude at maximum mesh displacement and final iteration.

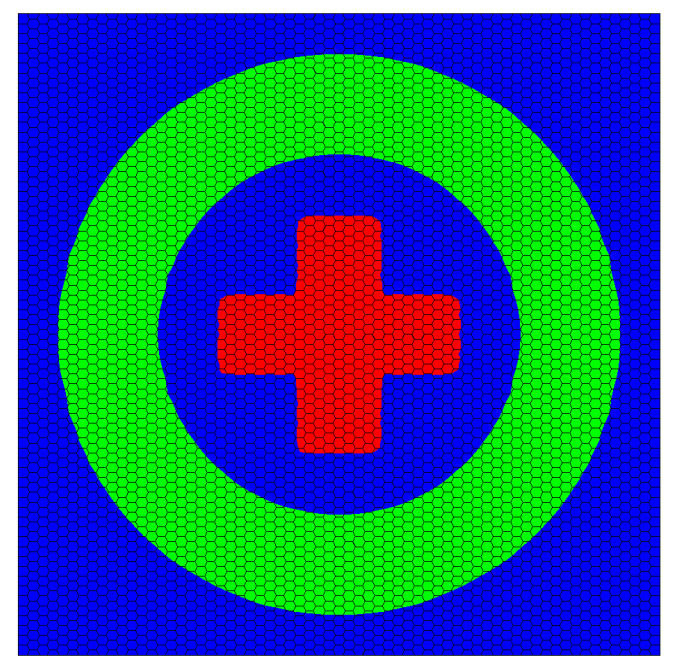

Figure 12: Multimaterial cyclic remap: materials on a honeycomb mesh.

For this challenging variant of the cyclic remap, we report linear convergence in total zone density and tangential velocity and sub-linear convergence 

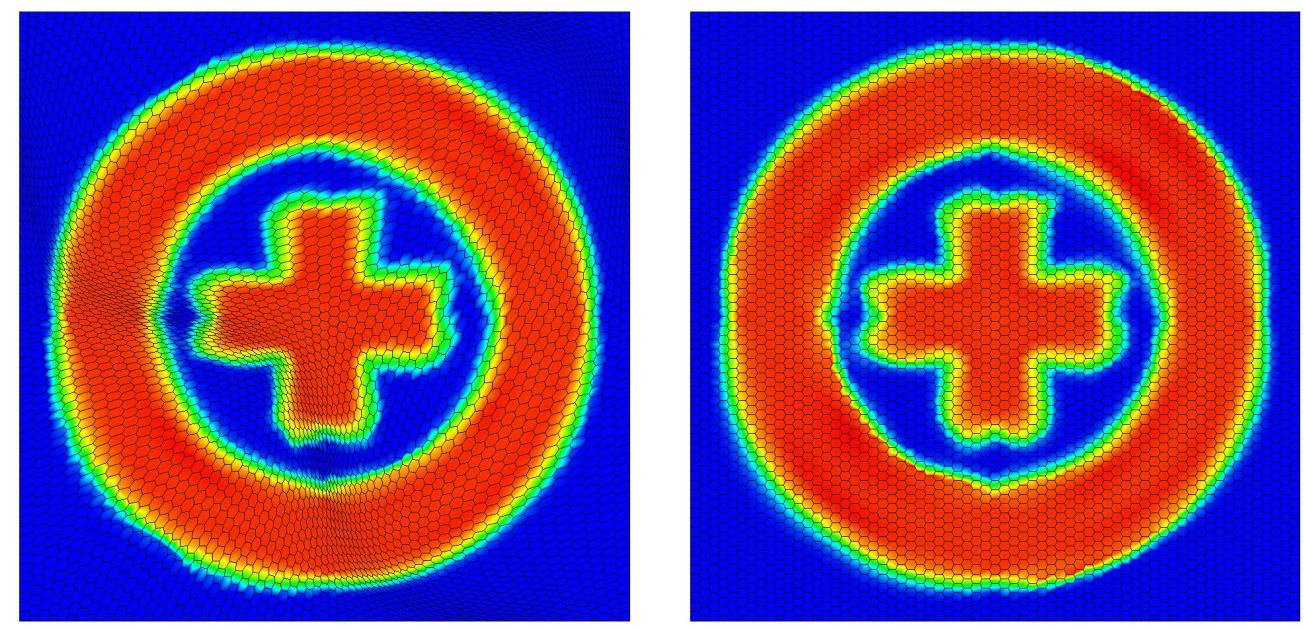

Figure 13: Multimaterial cyclic remap: velocity magnitude on a honeycomb mesh at maximum displacement (left) and final iteration (right).

in total node density, radial velocity, and reconstructed material-dependent subzonal densities. Convergence rates are summarized in fig. 14. Nodal density repair is turned on in this example.

\subsection{Hydrodynamic Tests}

We consider two multi-component ALE hydrodynamics tests: the triple point problem and a slab impactor problem. Each simulation cycle consists of a Lagrangian update followed by an overlay remap. The reconstruction algorithm is applied at every cycle to transform post-remap zone densities, node densities, and material volume fractions into consistent and conservative material-dependent subzonal densities.

\subsubsection{Eulerian Triple Point}

First is the triple point test. This is a $2 \mathrm{D}$ multi-component shock tube problem. A vertical planar interface separates a high-pressure, high-density gas on the left from two low-pressure gases having different densities on the right. A horizontal planar interface separates the two low-pressure gases. Figure 15 details the initial state variables and problem dimensions used. We use the same initial values and base mesh resolution as [14].

The pressure jump across the vertical interface induces a strong rightmoving shock into the low-pressure gases. The shock travels faster in the 

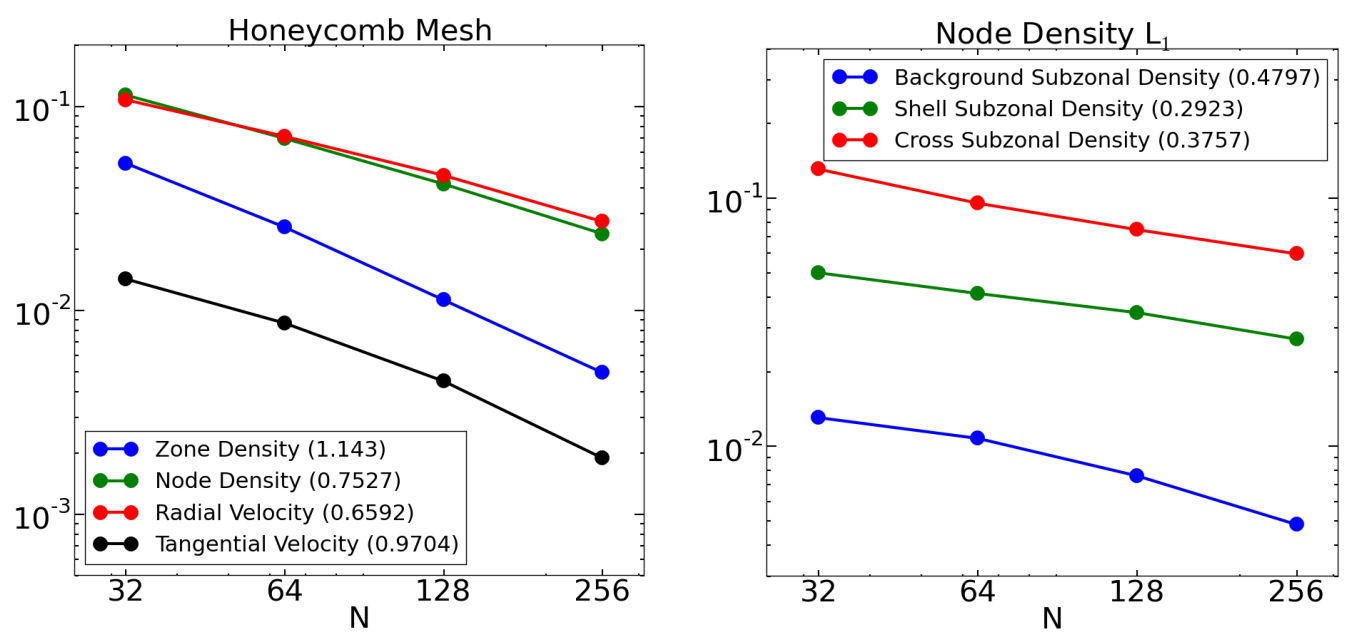

Figure 14: Multimaterial cyclic remap: $L_{1}$ convergence in remapped quantities (left) and reconstructed quantities (right) for honeycomb mesh of approximately $N^{2}$ elements.

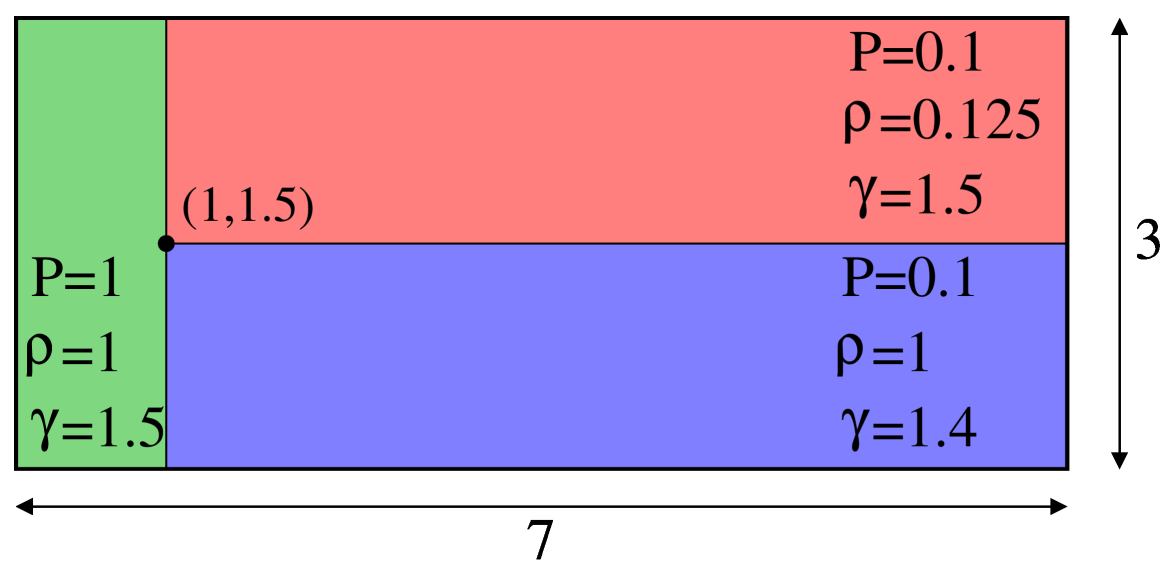

Figure 15: Triple Point test problem dimensions and initial conditions for pressure $P$, density $\rho$, and adiabatic index $\gamma$.

higher-density gas than in the lower-density one and produces shear along the horizontal interface separating them. The shear produces Kelvin-Helmholtzlike roll-up along the horizontal interface leading to a region of high vorticity near the initial material triple junction. We run the problem to $t=7$, at 
which time the shock front has reflected off the far right wall of the domain and interacts with the roll-up region.

We note that the triple point problem lacks a converged solution. No physical dissipation exists in the gas dynamics equations to damp the growth of structure along the unstable material interfaces. The amount of interfacial structure observed in the roll-up region of the numerical solution increases under grid refinement. The growth of structure is limited by the numerical dissipation of the hydro solver as well as the inherent resolution of the mesh to represent the structure.

We treat the triple point problem as a qualitative efficacy test of the reconstruction algorithm and present its self-convergence in the Eulerian frame with respect to a numerical solution at high mesh resolution. We select $70 \times 30$ as our base " $1 \mathrm{x}$ " mesh resolution and use the " $8 \mathrm{x}$ " mesh at $560 \times 240$ as the reference solution.

Figure 16 gives the energy profile at time $t=3.4$ for four different mesh resolutions. This time corresponds approximately to when the shock traveling through the top material strikes the right domain boundary. Note the amount of interface roll-up increases as the grid spacing decreases. This is attributable to decreases in numerical dissipation and increases in grid resolution of fine-scale structure.
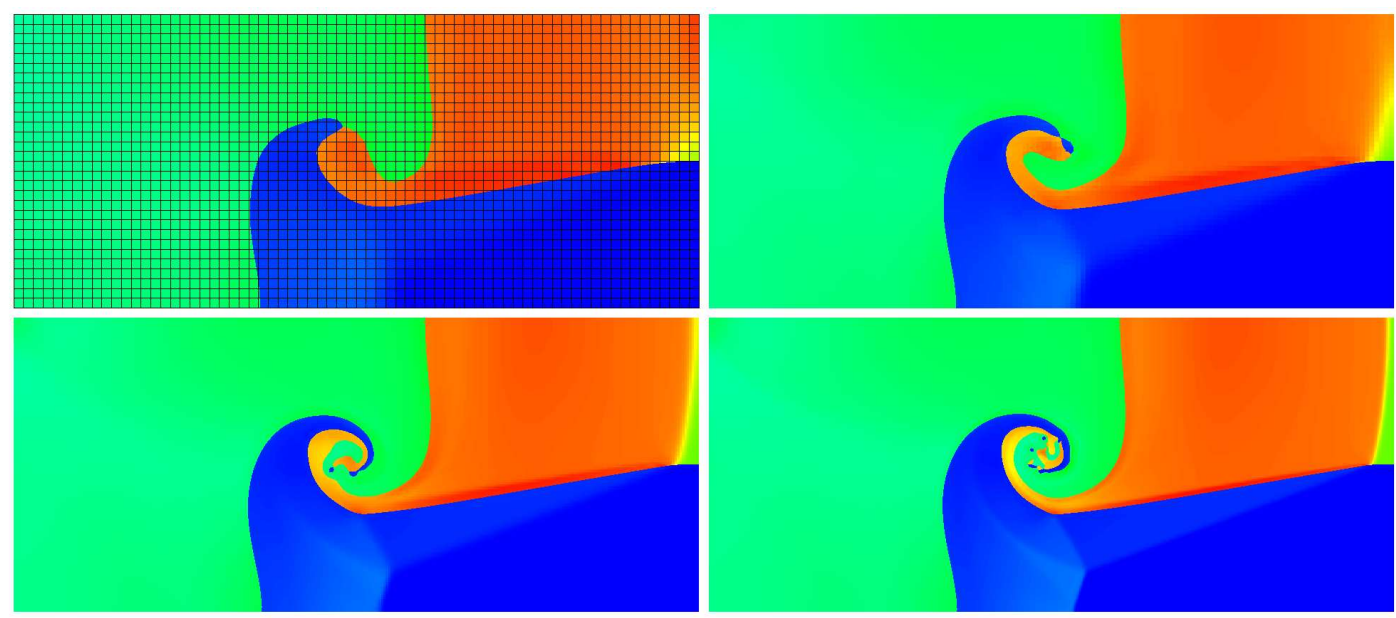

Figure 16: Eulerian Triple Point test: internal energy at $t=3.4$. Mesh resolutions $70 \times 30$ (top left), $140 \times 60$ (top right), $280 \times 120$ (bottom left), and $560 \times 240$ (bottom right). 
Figure 17 and fig. 18 show similar trends at $t=5$ and final time $t=7$ respectively. Time $t=5$ corresponds to the collision of the reflected shock with the initial vertical interface; time $t=7$ corresponds to the final interaction between the reflected shock and the roll-up region.
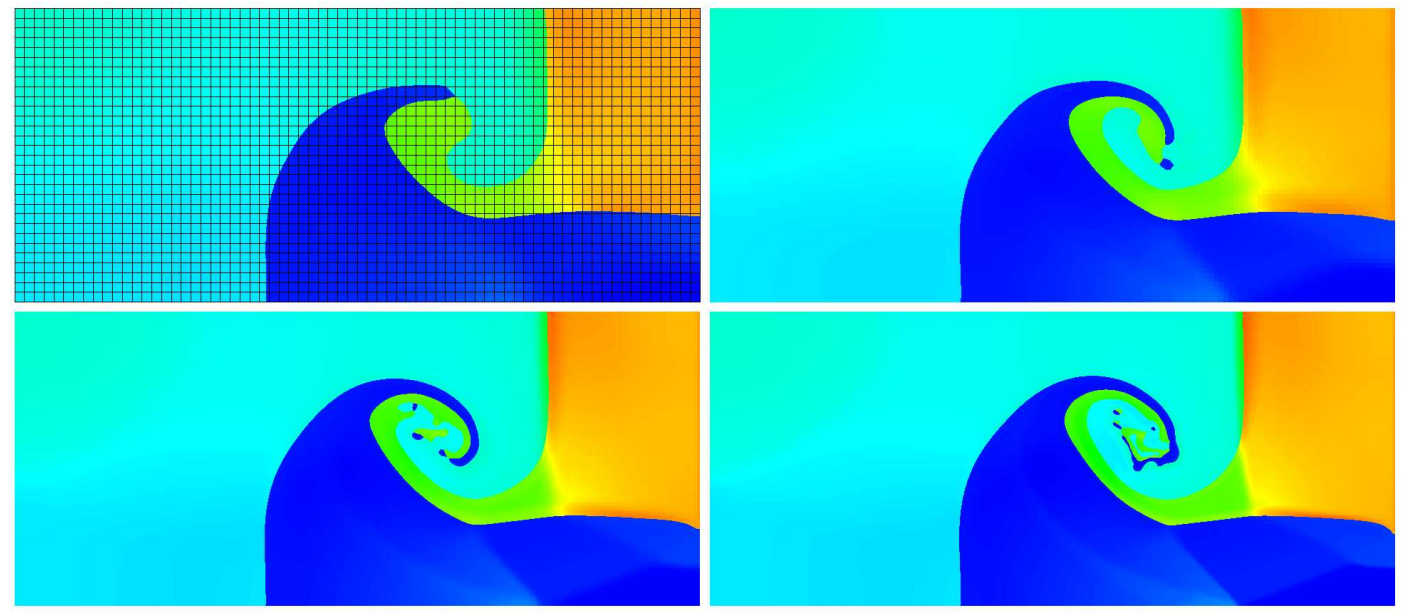

Figure 17: Eulerian Triple Point test: internal energy at $t=5$. Mesh resolutions $70 \times 30$ (top left), $140 \times 60$ (top right), $280 \times 120$ (bottom left), and $560 \times 240$ (bottom right).

Although convergence with respect to an analytic solution is not possible, we can nevertheless show that our methodology is consistent. Figure 19 establishes self-convergence in $L_{1}$ with respect to a highly-resolved reference solution. Convergence is close to first order - the maximum allowable for this shock-dominated problem-measured in zone density, internal energy, pressure, and velocity.

\subsubsection{ALE Impact Test}

Our second example tests the multimaterial reconstruction algorithm in the context of ALE hydrodynamics with geometric remapping. We consider the high-velocity impact problem introduced in [4] and used by [17] to test their conservative flux-based remap strategy. The problem consists of a high-density, high-velocity impactor striking a stationary, lower-density slab within a lowest-density background material. The strong shocks and deformations resulting from this configuration benefit greatly from the ALE methodology; Lagrangian calculations lead to tangled mesh elements that 

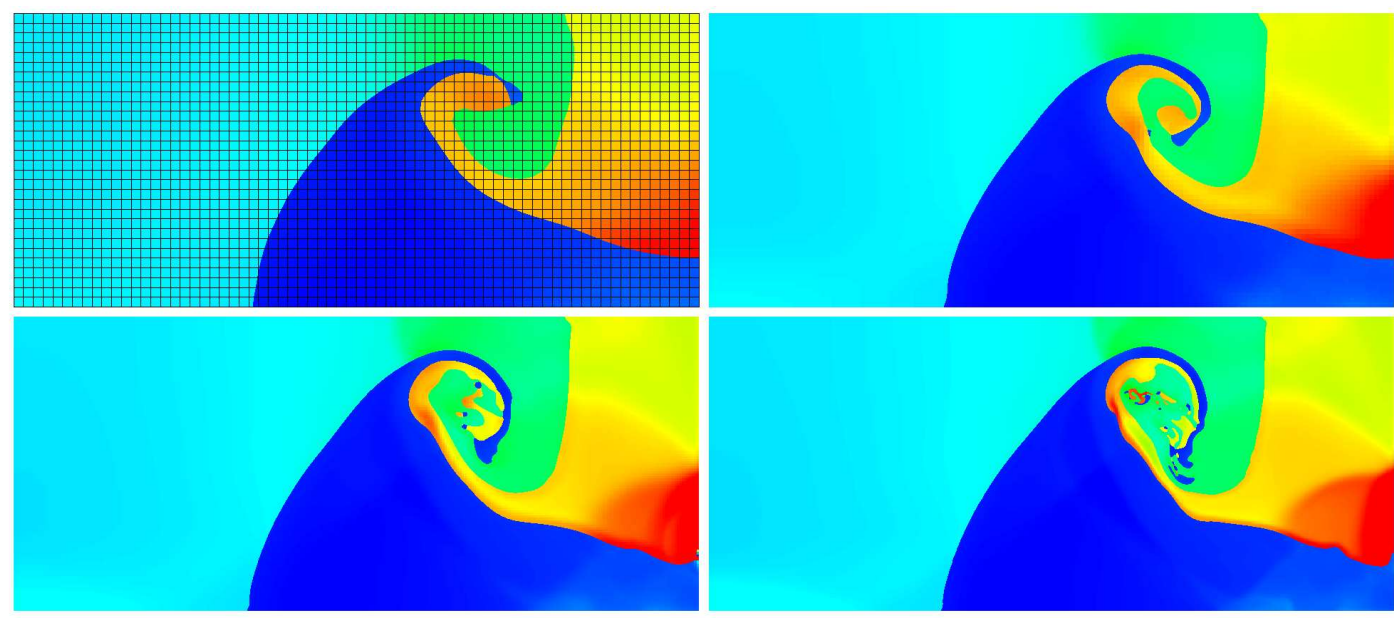

Figure 18: Eulerian Triple Point test: internal energy at final time $t=7$. Mesh resolutions $70 \times 30$ (top left), $140 \times 60$ (top right), $280 \times 120$ (bottom left), and $560 \times 240$ (bottom right).

ultimately terminate the simulation, while Eulerian-frame calculations tend to excessively diffuse flow information and are overly sensitive to interface reconstruction strategies.

\begin{tabular}{lllllllll}
\hline Material & $\gamma$ & $\rho$ & $P$ & $v_{x}$ & $x_{\min }$ & $x_{\max }$ & $y_{\min }$ & $y_{\max }$ \\
\hline Background & 1.4 & 1.0 & 1.0 & 0.0 & 0.0 & 2.0 & 0.0 & 2.0 \\
Impactor & 50.0 & 20.0 & 2.0 & 0.2 & 0.25 & 0.75 & 0.9 & 1.1 \\
Slab & $5.0 / 3$ & 15.0 & 1.0 & 0.0 & 1.0 & 1.1 & 0.0 & 2.0 \\
\hline
\end{tabular}

Table 4: Initial conditions for the high-velocity impact test. Columns indicate ideal gas index $\gamma$, density $\rho$, pressure $P, x$ velocity $v_{x} .\left(x_{\min }, x_{\max }\right)$ and $\left(y_{\min }, y_{\max }\right)$ determine the initial bounding regions of the materials.

Table 4 summarizes the initial setup for the impact test in terms of density, pressure, and velocity. Each material is modeled as an ideal gas with index $\gamma$. Reflecting boundary conditions are specified at the each side of the domain $\{(x, y) \in[0,2] \times[0,2]\}$. We simulate this problem on regular $N \times N$ meshes for $N \in\{80,160,320,640\}$ to final time $t=8$. We prescribe an identical mesh relaxation strategy to [17], whereby nodes are adaptively flagged 


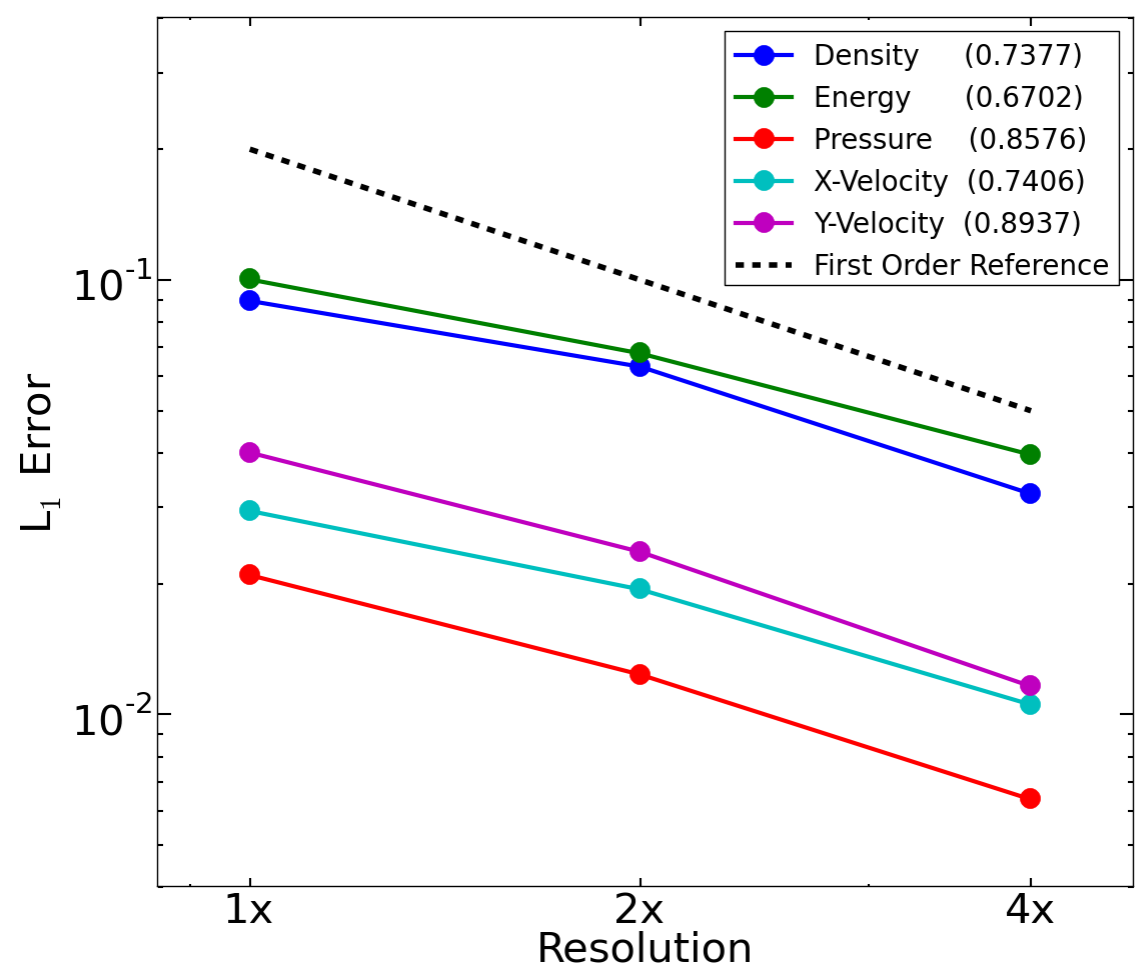

Figure 19: Eulerian Triple Point test: self-convergence of solutions at $t=7$ for resolutions $70 \times 30,140 \times 60$, and $280 \times 120$ with respect to a reference solution on $560 \times 240$ mesh.

for relaxation by thresholds on various local metrics related to zone shape.

Figure 20 illustrates the numerical solution at time $t=4$ for the four mesh resolutions. Similarly, fig. 21 gives the numerical solution at the final time. Qualitatively, our solution is in good agreement with those presented in [17], with small differences in the shape and location of small, under-resolved material islands and filaments. The two simulations use identical Lagrangian hydrodynamics algorithms, mesh relaxation strategies, and relaxers. Where they differ is in interface reconstruction and remapping algorithms. It is therefore not surprising that the macroscopic solutions are so similar and that differences occur at interfaces on the smallest resolvable scale.

For a problem such as this one involving strong, reflecting shocks and 

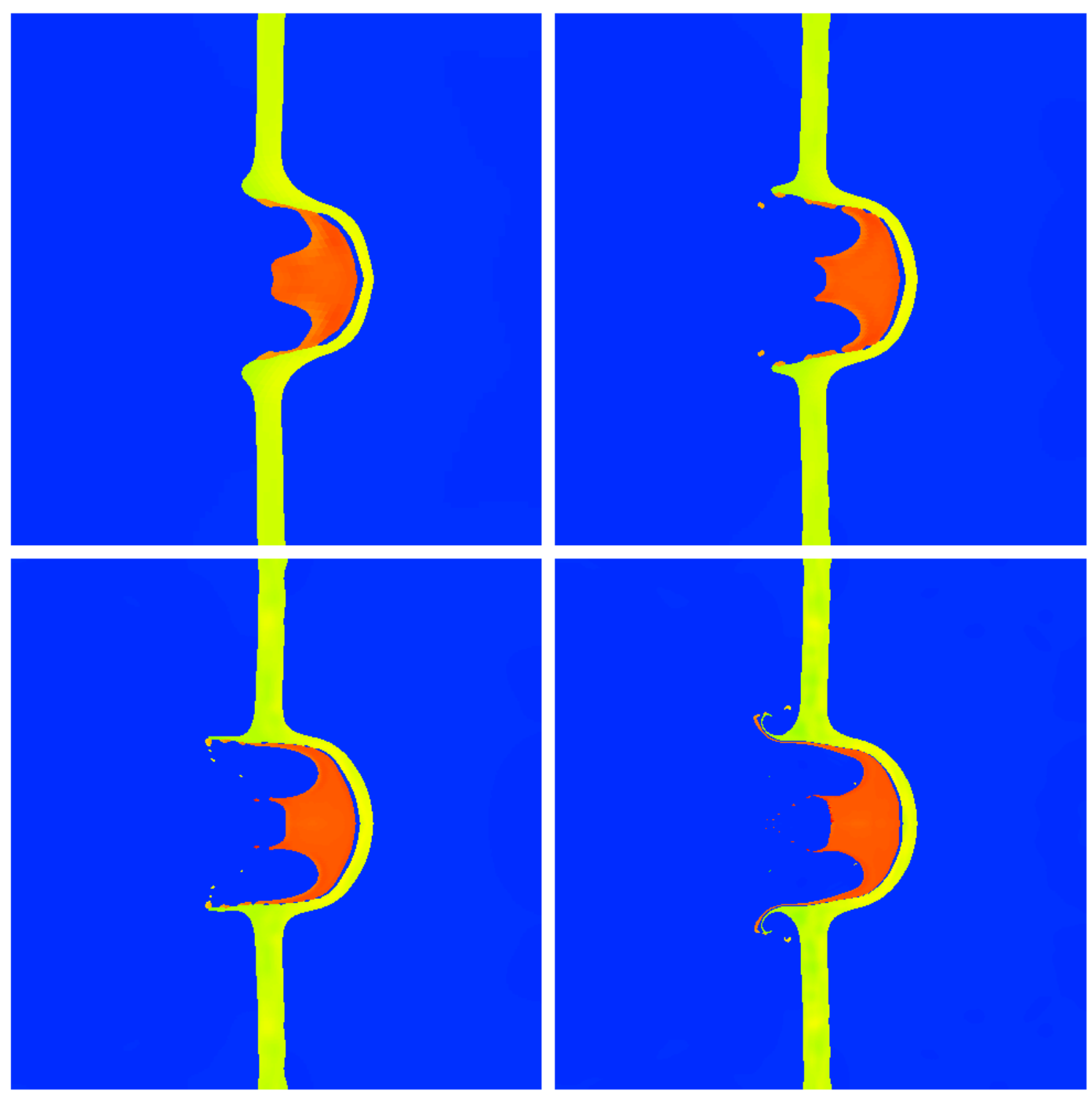

Figure 20: Material distribution in the ALE impact test at $t=4$. The four panels show meshes consisting of $80^{2}$ zones (upper left), $160^{2}$ zones (upper right), $320^{2}$ zones (lower left), and $640^{2}$ zones (lower right).

unstable material interfaces, a grid-converged solution is not available. As a means of measuring solution convergence quantitatively, we establish selfconvergence with respect to various quantities over time. Following [17], our 

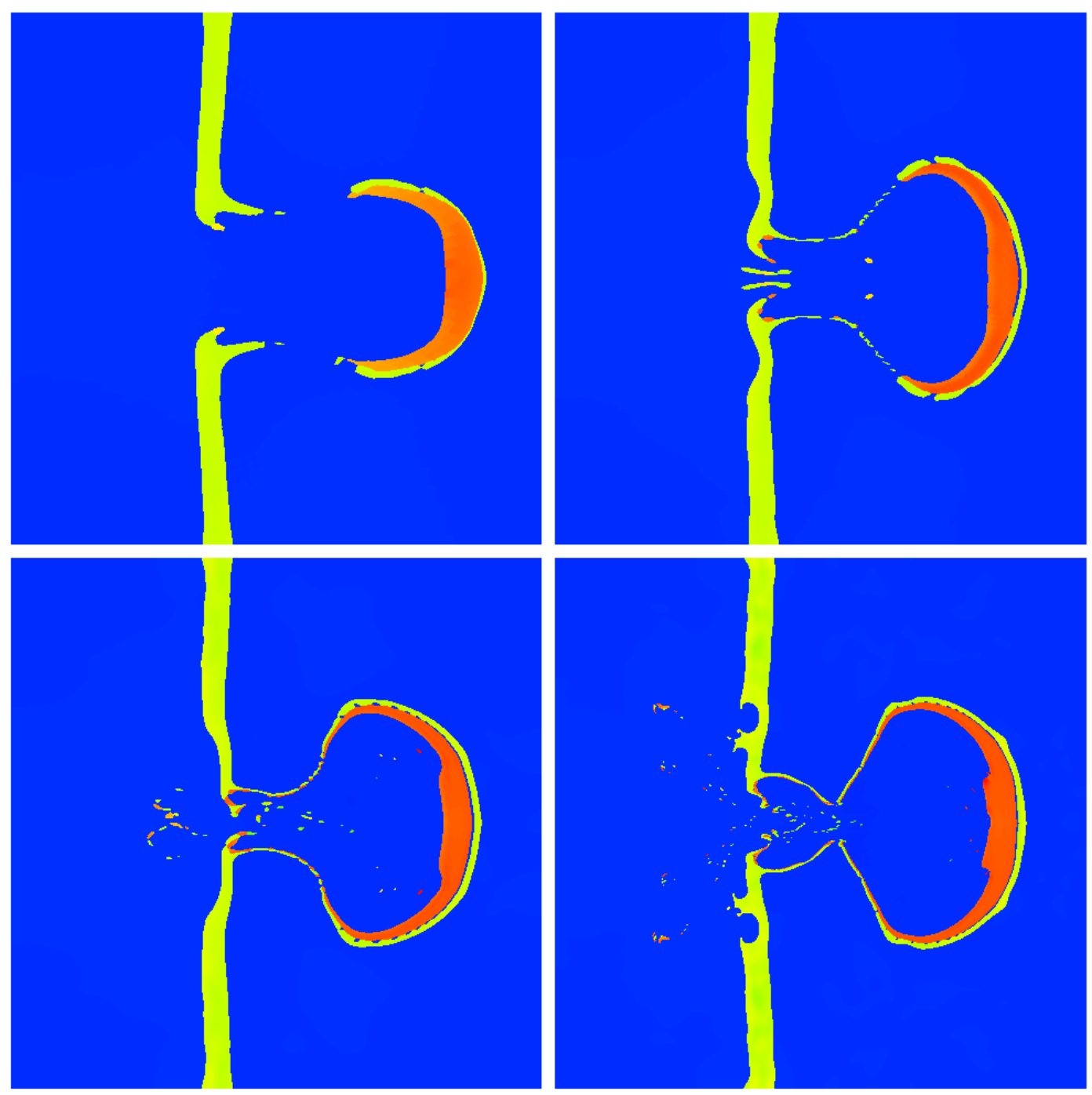

Figure 21: Material distribution in the ALE impact test at $t=8$. The four panels show meshes consisting of $80^{2}$ zones (upper left), $160^{2}$ zones (upper right), $320^{2}$ zones (lower left), and $640^{2}$ zones (lower right).

first metric involves the $x$-velocity of the impactor. At each cycle, we compute the mass centroid of the impactor material and report the $x$-component of velocity at that point. Figure 22 gives the velocity at each cycle for the four 
mesh resolutions. The convergence trend matches that of [17].

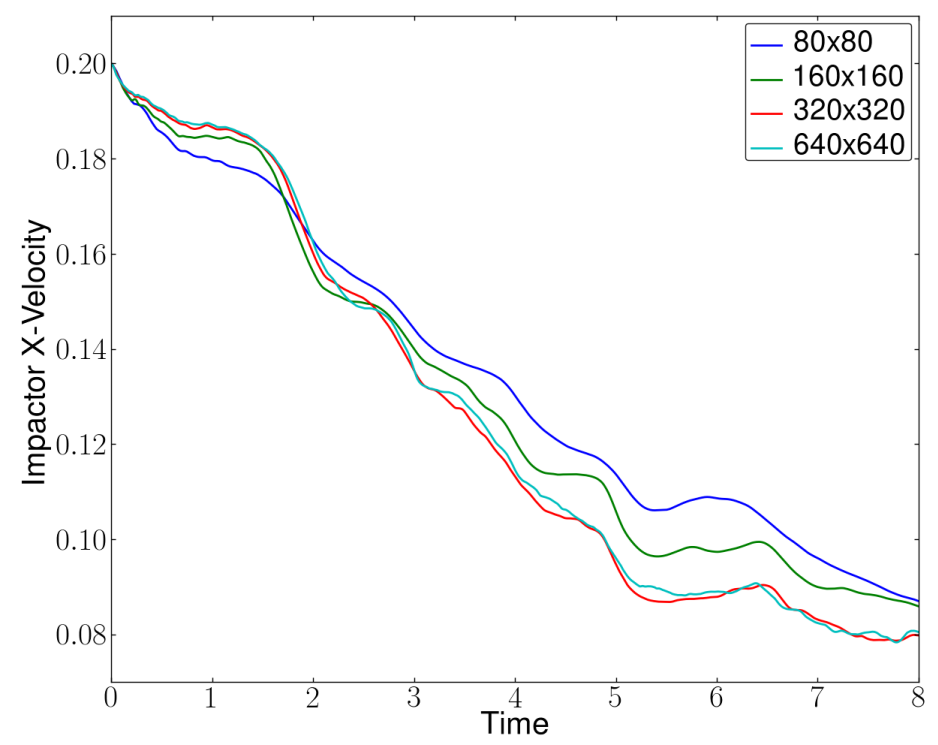

Figure 22: Time history of the center of mass $x$ velocity in the impact ALE test at four different mesh resolutions.

For the second and third metric, we consider the ejected mass of the impactor and slab respectively. We roughly define material as being "ejected" if it is located to the right of $x_{\max }^{s l a b}=1.1$. The ejected mass is initially zero for the two materials. At the final time, most of the impactor has passed through the slab; some percentage remains behind the slab due to the extreme deformations in both the slab and impactor. Only a small percent of the total slab mass is ejected. The slab itself recoils when struck by the impactor and travels left-ward; the amount of slab mass that is ejected corresponds to the thin, deformed filamentary region pushed outward by the impactor itself. Figure 23 shows the time history of the ejected mass percentages (impactor on the left and slab on the right). The impactor converges to about $85 \%$ of total mass, and the slab to approximately $21 \%$.

Figure 24 illustrates the self-convergence trends in time-integrated $L_{1}$ error for each of the three metrics. Error is computer relative to the most resolved calculation. Linear fits to the data report self-convergence between 

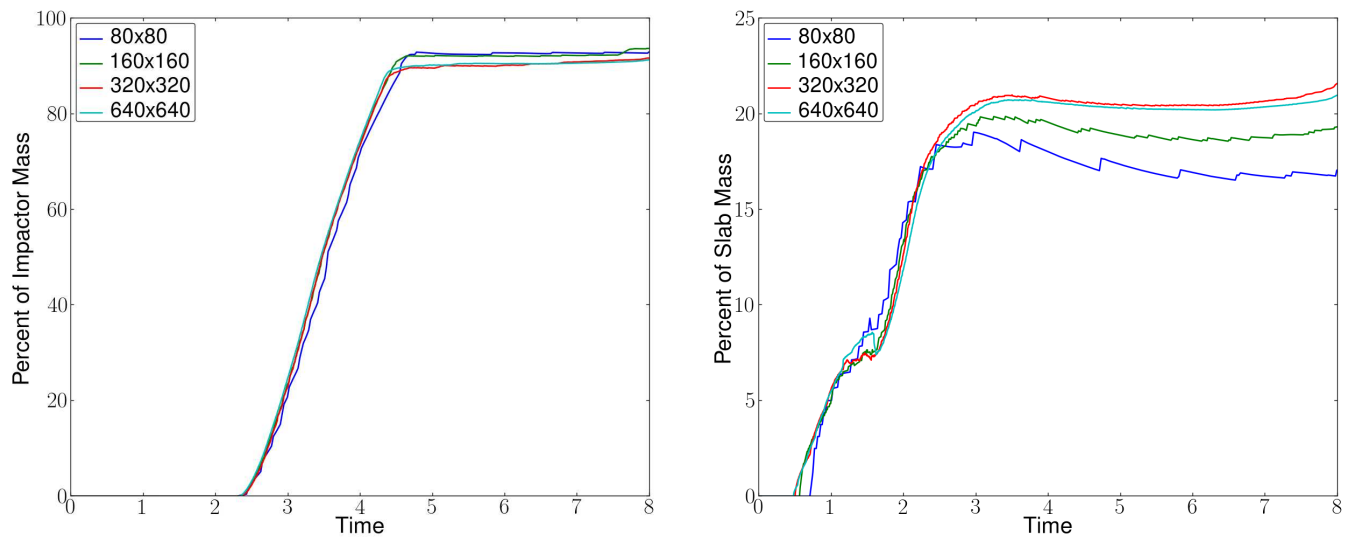

Figure 23: Time history of the percentage of the mass with $x>1.1$ in the ALE impact test case. The left panel shows the evolution of the impactor material, and the right the slab.

first and second order. Note that for the two mass metrics, we compute $L_{1}$ error in units of mass, not percentage of total material mass. This is purely for the convenience of plotting all three metrics on the same image.

\section{Conclusions}

We have developed a multi-material extension to the subzonal reconstruction algorithm in [23]. Reconstruction can be interpreted as a post-processing step on overlay-based remap data to restore sub-grid information and thus momentum conservation. The multi-material extension can be thought of as a preprocessing or preconditioning step computed after the overlay but before reconstruction. After reconstruction, we assign material mass to each subzone such that the subzonal mass fraction equals the mass fraction of the zone that contains it. The multimaterial extension is fully conservative and trivially simplifies to the original reconstruction algorithm if only one material is present.

Numerical results are very favorable. For pure-remapping tests, the algorithm reconstructs material interface data for both discontinuous and smoothed profiles. Grid convergence is established on quad and polygonal meshes. Further, the accuracy of the reconstructed data appears insensitive to the quality of the target mesh. 


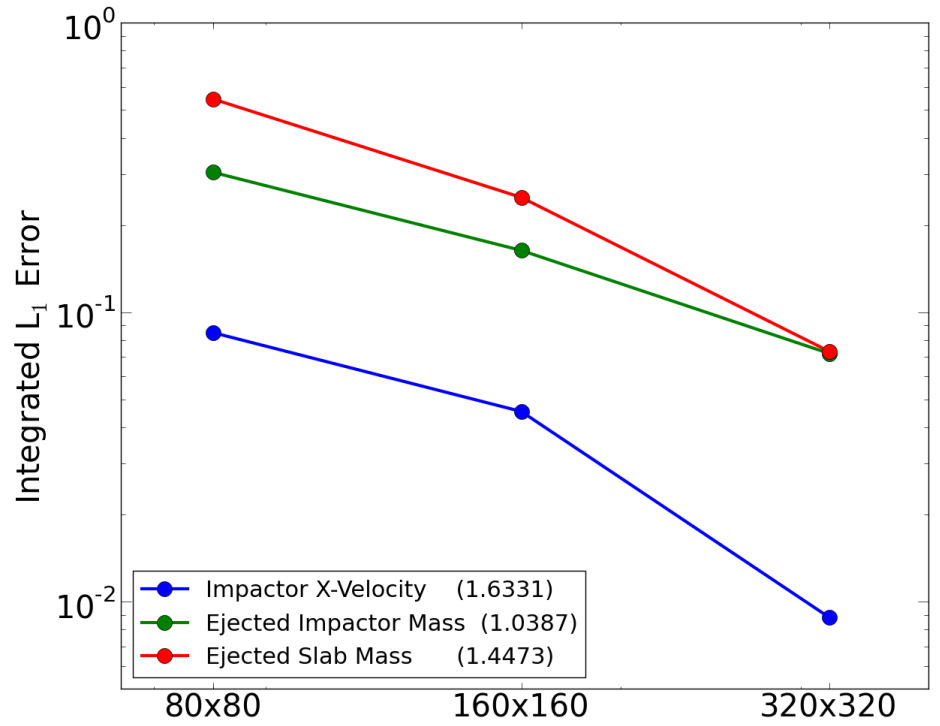

Figure 24: Self-convergence rates for the ALE impact slab test case.

For ALE hydro problems, we demonstrate the robustness of the new algorithm on tests having steep density discontinuities, highly vortical flows, and large grid distortions. Unfortunately, the problems of interest - trulymultimaterial gas dynamics in two dimensions - lack known solutions. Grid convergence to an analytic profile is not assured. Instead, we demonstrate self-convergence of our multi-material ALE hydro solver with subzonal reconstruction to a reference solution (computed with the same solver) on a highly-resolved grid.

The key component of the multimaterial extension is the preprocessing step. If post-remap nodal mass is not corrected to account for interface reconstruction, then zone and node mass can become inconsistent. This can lead to large monotonicity errors in the reconstructed subzonal density field. This leads to negative subzonal values that require correcting, ultimately resulting in momentum conservation errors over time.

The preprocessing step developed here is compatible with the overlay remapping tools available to us. We admit that a better treatment is indeed possible for correcting nodal mass. One possible direction involves combin- 
ing additional information about intersection volumes and material interface locations to compute discrete mass fluxes interior to the target mesh zones. The work of Kucharik and Shashkov in [11] provides an excellent framework for how to proceed.

\section{Acknowledgments}

This work was performed under the auspices of the U. S. Department of Energy by Lawrence Livermore National Laboratory under Contract DEAC52-07NA27344.

\section{References}

[1] P. Anninos, Kull ALE: I. Unstructured Mesh Advection, Interface Capturing, and Multiphase 2T RHD with Material Interfaces, Technical Report: UCRL-ID-147297-PT-1, Lawrence Livermore National Laboratory, 2002.

[2] P. Anninos, Kull ALE: II. Grid Motion on Unstructured Arbitrary Polyhedral Meshes, Technical Report: UCRL-ID-147297-PT-2, Lawrence Livermore National Laboratory, 2002.

[3] P. Anninos, New VOF Interface Capturing and Reconstruction Algorithms, Technical Report: UCRL-ID-135084, Lawrence Livermore National Laboratory, 1999.

[4] A. Barlow, R. Hill, M. Shashkov, Constrained Optimization Framework for Interface-Aware Sub-Scale Dynamics Closure Models for Multimaterial Cells in Lagrangian and Arbitrary Lagrangian-Eulerian Hydrodynamics, Journal of Computational Physics, LA-UR-13-26180, 2014. Submitted for publication.

[5] E. Caramana, D. Burton, M. Shashkov, P. Whalen, The Construction of Compatible Hydrodynamics Algorithms Utilizing Conservation of Total Energy, Journal of Computational Physics, 146, pp. 227-262, 1998.

[6] J. Grandy, Conservative Remapping and Region Overlays by Intersecting Arbitrary Polyhedra, Journal of Computational Physics, 148, pp. 433-466, 1999. 
[7] hypre. High performance preconditioners. http://www.llnl.gov/CASC/linearsolvers

[8] J. Johnson, J. M. Owen, D. Starinshak (2014), Polytope. https://bitbucket.org/jjphatt/polytope.

[9] W. Karush, Minima of Functions of Several Variables with Inequalities as Side Constraints, MS Dissertation, Department of Mathematics, University of Chicago, 1939.

[10] M. Kucharik, J. Breil, S. Galera, P.-H. Maire, M. Berndt, M. Shashkov, Hybrid Remap for Multi-Material ALE, Computers and Fluids, 46, pp. 293-297, 2010.

[11] M. Kucharik and M. Shashkov, Conservative Multi-Material Remap for Staggered Multi-Material Arbitrary Lagrangian-Eulerian Methods, Journal of Computational Physics, 256, pp. 268-304, 2014.

[12] H. Kuhn and A. Tucker, Nonlinear Programming, Proceedings of 2nd Berkeley Symposium on Mathematical Statistics and Probability, pp. 481-492, 1951.

[13] R. Liska, M. Shashkov, P. Vachal, B. Wendroff, Optimization-Based Synchronized Flux-Corrected Conservative Interpolation (Remapping) of Mass and Momentum for Arbitrary Lagrangian-Eulerian Methods, Journal of Computational Physics, 229, pp. 1467-1497, 2010.

[14] R. Loubere, P.-H. Maire, M. Shashkov, J. Breil, S. Galera, ReALE: A Reconnection-Based Arbitrary-Lagrangian-Eulerian Method, Journal of Computational Physics, 229, pp. 4724-4761, 2010.

[15] R. Loubere and M. Shashkov, A Subcell Remapping Method on Staggered Polygonal Grids for Arbitrary-Lagrangian-Eulerian Methods, Journal of Computational Physics, 209, pp. 105-138, 2005.

[16] L. Margolin and M. Shashkov, Remapping Recovery and Repair on a Staggered Grid, Comput. Methods Appl. Engrg., 193, pp. 4139-4155, 2004.

[17] J. M. Owen, M. Shashkov, Arbitrary Lagrangian Eulerian Remap Treatments Consistent with Staggered Compatible Total Energy Conserving 
Lagrangian Methods, Journal of Computational Physics, 273, pp. 520$547,2014$.

[18] J. Pilliod, An Analysis of Piecewise Linear Interface Reconstruction Algorithms for Volume-of-Fluid Methods, M. S. Thesis, University of California, Davis, September 1992.

[19] J. Rathkopf, D. Miller, J. M. Owen, L. Stuart, M. Zika, P. Eltgroth, N. Madsen, K. McCandless, P. Nowak, M. Nemanic, N. Gentile, N. Keen, T. Palmer Kull: LLNL's ASCI Inertial Confinement Fusion Simulation Code.

[20] L. Sedov, Propagation of Strong Shock Waves, Journal of Applied Mathematics and Mechanics, v. 10, pp. 241-250, 1946.

[21] L. Sedov, Similarity and Dimensional Methods in Mechanics, Academic Press, New York, 1959.

[22] J Lockwood-Taylor, An Exact Solution of the Spherical Blast Wave Problem, Philosophical Magazine, v. 46, pp. 317-320, 1955.

[23] D. Starinshak, J. M. Owen, A Subzone Reconstruction Algorithm for Staggered Compatible Remapping, Journal of Computational Physics, 296, pp. 263-292, 2015. 